\title{
Multi-fibre optical spectroscopy of low-mass stars and brown dwarfs in Upper Scorpius ${ }^{\star \star \star \star, \star \star \star}$
}

\author{
N. Lodieu ${ }^{1,2}$, P. D. Dobbie ${ }^{3}$, and N. C. Hambly ${ }^{4}$ \\ 1 Instituto de Astrofísica de Canarias (IAC), Calle Vía Láctea s/n, 38200 La Laguna, Tenerife, Spain \\ e-mail: nlodieu@iac.es \\ 2 Departamento de Astrofísica, Universidad de La Laguna (ULL), 38205 La Laguna, Tenerife, Spain \\ 3 Australian Astronomical Observatory, PO Box 296, Epping, NSW, 1710, Australia \\ e-mail: pdd@aao.gov. au \\ 4 Scottish Universities' Physics Alliance (SUPA), Institute for Astronomy, School of Physics, University of Edinburgh, \\ Royal Observatory, Blackford Hill, Edinburgh EH9 3HJ, UK \\ e-mail: nch@roe.ac.uk
}

Received 14 May 2010 / Accepted 8 December 2010

\section{ABSTRACT}

\begin{abstract}
Context. Knowledge of the mass function in open clusters constitutes one way to critically examine the formation mechanisms proposed to explain the existence of low-mass stars and brown dwarfs.

Aims. The aim of the project is to determine as accurately as possible the shape of the mass function across the stellar/substellar boundary in the young $(5 \mathrm{Myr})$ and nearby $(d=145 \mathrm{pc})$ Upper Sco association.

Methods. We have obtained multi-fibre intermediate-resolution $(R \sim 1100)$ optical $(\sim 5750-8800 \AA)$ spectroscopy of 94 photometric and proper motion selected low-mass star and brown dwarf candidates in Upper Sco with the AAOmega spectrograph on the AngloAustralian Telescope.

Results. We have estimated the spectral types and measured the equivalent widths of youth ( $\mathrm{H} \alpha$ ) and gravity (Na I and K I) diagnostic features to confirm the spectroscopic membership of about $95 \%$ of the photometric and proper motion candidates extracted from 6.5 square degrees surveyed in Upper Sco by the UKIRT Infrared Deep Sky Survey (UKIDSS) Galactic Clusters Survey (GCS). We also detect lithium in the spectra with the highest signal-to-noise, consolidating our conclusions about their youth. Furthermore, we derive an estimate of the efficiency of the photometric and proper motion selections used in our earlier studies using spectroscopic data obtained for a large number of stars falling into the instrument's field-of-view. We have estimated the effective temperatures and masses for each new spectroscopic member using the latest evolutionary models available for low-mass stars and brown dwarfs. Combining the current optical spectroscopy presented here with near-infrared spectroscopy obtained for the faintest photometric candidates, we confirm the shape and slope of our earlier photometric mass function. The luminosity function drawn from the spectroscopic sample of 113 USco members peaks at around M6 and is flat at later spectral type. We may detect the presence of the M7/M8 gap in the luminosity function as a result of the dust properties in substellar atmospheres. The mass function may peak at $0.2 M_{\odot}$ and is quite flat in the substellar regime. We observe a possible excess of cool low-mass brown dwarfs compared to IC 348 and the extrapolation of the field mass functions, supporting the original hypothesis that Upper Sco may possess an excess of brown dwarfs compared to other young regions.

Conclusions. This result shows that the selection of photometric candidates based on five band photometry available from the UKIDSS GCS and complemented partially by proper motions can lead to a good representation of the spectroscopic mass function.
\end{abstract}

Key words. techniques: spectroscopic - open clusters and associations: individual: Upper Scorpius - brown dwarfs stars: luminosity function, mass function

\section{Introduction}

Firm knowledge of the form of the initial mass function i.e. the number of objects as a function of mass (IMF, Salpeter 1955; Miller \& Scalo 1979; Scalo 1986) is vital to understanding the formation of low-mass stars and brown dwarfs. To address fundamental issues like the universality of the IMF (or

* Based on observations obtained with the AAOmega spectrograph at the Anglo-Australian Observatory.

$\star \star$ Appendices are only available in electronic form at http://www. aanda.org

$\star \star \star$ Full Table B.1 and optical spectra are only available in electronic form at the CDS via anonymous ftp to

cdsarc.u-strasbg.fr $(130.79 .128 .5)$ or via

http://cdsarc.u-strasbg.fr/viz-bin/qcat?J/A+A/527/A24 otherwise its dependence with environment, time and/or any other factor), masses and effective temperatures $\left(T_{\text {eff }}\right)$ must be estimated as accurately as possible for all members in open clusters and star-forming regions. The advent of large optical charge-coupled devices (CCDs) and infrared detectors has facilitated the study of numerous regions with different ages, densities, and histories. However, the samples of photometric candidates confirmed spectroscopically in those regions for an unbiased estimate of the IMF over a large mass range remains limited although these are growing rapidly. Complete spectroscopic mass functions over the full mass range probed by the associated photometric surveys have been presented in the central region of the Trapezium Cluster by Luhman et al. (2000) and Slesnick et al. (2004) and compared to photometric estimates (Hillenbrand \& Carpenter 2000; Muench et al. 2002). 
Similarly, the Taurus region and the IC 348 cluster have been targeted photometrically and spectroscopically down to the substellar regime (Luhman 2000; Briceño et al. 2002; Luhman et al. 2003a,b). More recently, Luhman (2007) updated the census of low-mass stars and brown dwarfs in Chamaeleon I star-forming region based on optical and near-infrared spectroscopy. The derived mass function in those regions would suggest a dependence on environment of the IMF since the peak in the luminosity function of Taurus is at higher masses than in the Trapezium Cluster and IC 348 (0.8 vs. 0.1-0.2 $M_{\odot}$, Luhman 2004b).

The Upper Sco association (hereafter USco) is part of the Scorpius Centaurus OB association: it is located at $145 \mathrm{pc}$ from the Sun (de Bruijne et al. 1997) and its age is estimated to be $5 \pm 2$ Myr from isochrone fitting and dynamical studies (Preibisch \& Zinnecker 2002). The association was targeted in X-rays (Walter et al. 1994; Kunkel 1999; Preibisch et al. 1998), with Hipparcos (de Bruijne et al. 1997; de Zeeuw et al. 1999), and more recently at optical (Preibisch et al. 2001; Preibisch \& Zinnecker 2002; Ardila et al. 2000; Martín et al. 2004; Slesnick et al. 2006) and near-infrared (Lodieu et al. 2006, 2007b) wavelengths. As a result of the latest surveys, several tens of brown dwarfs were confirmed spectroscopically as members of the association (Martín et al. 2004; Slesnick et al. 2006; Lodieu et al. 2006; Slesnick et al. 2008; Lodieu et al. 2008; Martin et al. 2010), resulting in a total of 92 substellar members divided into 76 M6-M9, and 16 L0-L2 dwarfs. Slesnick et al. (2008) presented the first spectroscopic mass function in USco over the full area of the association down to $M=0.02 M_{\odot}$.

In this paper we present follow-up multi-fibre optical spectroscopy of low-mass star and brown dwarf candidates extracted from 6.5 square degrees targeted by the UKIRT Infrared Deep Sky Survey (UKIDSS, Lawrence et al. 2007) Galactic Clusters Survey (GCS) and published in Lodieu et al. (2007b). In Sect. 2 we define the various samples of targets included in our spectroscopic follow-up. In Sect. 3, we describe the spectroscopic observations made with the AAOmega instrument installed on the 3.9-m Australian Astronomical telescope (AAT) and the data reduction. In Sect. 4 we assess the membership of the photometric candidates based on their spectral type, their chromospheric activity, and the strength of gravity-sensitive features. A similar analysis is presented for a sample of photometric nonmembers to estimate the reliability of our original photometric and proper motion selections in Sect. 5. In Sect. 6 we derive effective temperatures, bolometric luminosities, and masses for all USco members and compare the spectroscopic luminosity and mass functions in the low-mass and brown dwarf regimes to previous studies in USco and other regions.

\section{The samples}

Our sample consists of three groups of objects: firstly photometric USco candidates selected from the UKIDSS GCS Science Verification (Lodieu et al. 2007b); secondly, a few known spectroscopic members falling in the instrument field-of-view and published in the literature (Martín et al. 2004; Slesnick et al. 2006), and, finally a sample of photometric non-members.

The first sample consists of USco photometric candidates selected from Lodieu et al. (2007b). Out of the original 129 candidates, 114 are brighter than $Z=17.5 \mathrm{mag}$, corresponding to masses in the range $0.6-0.03 M_{\odot}$ at the age and distance of USco. We were able to obtain spectra for 94 candidates out of 114, implying a spectroscopic completeness of $82 \%$. Table A.1 lists the main properties of all 90 candidates targeted with AAT/AAOmega and subsequently confirmed as spectroscopic members, including coordinates, $J$-band magnitudes ${ }^{1}$, equivalent widths for the $\mathrm{H} \alpha$ line and the $\mathrm{NaI}$ and K I doublets, spectral indices, spectral types, effective temperatures, luminosities, and masses. For the $\mathrm{Na} I$ and $\mathrm{K}$ I doublets we give the equivalent width of the doublet and not of each line that constitutes the doublet although they are resolved at the resolution of our spectra. For completeness, we have added to Table A.1 the 19 sources confirmed as spectroscopic members in the near-infrared with Gemini/GNIRS (Lodieu et al. 2008) and identified photometrically by Lodieu et al. (2007b) ${ }^{2}$. We should mention that five photometric candidates included in the AAOmega survey are common to our earlier near-infrared spectroscopic follow-up (Lodieu et al. 2008).

The second sample consists of five known spectroscopic members with spectral types estimated from optical data with an accuracy of half a subclass: DENIS1610-2212 (Martín et al. 2004), SCH1611-2217, SCH1612-2349, SCH1612-2338, and SCH1613-2305 (Slesnick et al. 2006). These five objects fell in the AAOmega field-of-view and serve as spectral templates for assigning spectral types to our candidates. Table 1 provides the full coordinates, $J$-band photometry, equivalent width measurements (in $\AA$ ) of the $\mathrm{H} \alpha$ emission line and the gravity sensitive doublets ( $\mathrm{NaI}$ and $\mathrm{KI}$ ), as well as spectral types and names as quoted in the literature. The previously known members are not plotted in Fig. 1 because no $Z$-band magnitudes are available for them as they lie outside the UKIDSS GCS field-of-view (see Fig. 4 in Lodieu et al. 2007b).

The third sample contains 624 sources we originally rejected as members of USco based on their location in various colourmagnitude diagrams (Fig. 5 of Lodieu et al. 2007b). Two subsamples were selected to match the magnitude range of the USco photometric candidates. First, a bright sample which consists of 269 sources with $Z=12-14.5$ mag and $Z-J$ colours redder than 0.7 mag (only 4 have $Z-J<0.7$ mag; Fig. 1). Second, a faint sample is made of 355 objects with $Z=14.5-17.5$ mag and selected to the right of a straight line going from $(Z-J, Z)=(0.8$, $14.5)$ to $(1.2,17.5)$. These objects are shown as crosses in Fig. 1.

\section{Spectroscopic follow-up of USco candidates}

\subsection{Spectroscopic observations}

We have obtained low-resolution multi-fibre optical spectroscopy of 94 photometric and proper motion selected low-mass star and brown dwarf candidates in USco as well as a sample of photometric non members (Sect. 2) with the AAT/AAOmega spectrograph (Lewis et al. 2002; Sharp et al. 2006) ${ }^{3}$. AAOmega has a two degree field-of-view which is sampled by 400 fibres that feed a two-armed spectrograph. The projected diameter on the sky of each fibre is 2 arcsec and their minimum separation is 30 arcsec (Miszalski et al. 2006). On the red arm we have employed the $385 \mathrm{R}$ grating to cover the wavelength range $5700-8800 \AA$ (the exact coverage depends slightly on the position of the fibre on the spectrograph slit) at a resolution of $R \sim 1350$. Spectra covering the wavelength range 3740-5720 ̊,

\footnotetext{
1 The full $Z Y J H K$ photometry is published in Lodieu et al. (2007b). 223 candidates were presented in Lodieu et al. (2008): 20 are from Lodieu et al. (2007b) and three from Lodieu et al. (2006). Two were classified as non members among the 20 candidates from Lodieu et al. (2007b) but one is now reclassified as a member after optical spectroscopy (see Sect. 4.5).

3 More details on the AAOmega spectrograph can be found at the URL: http://www . aao.gov . au/AAO/astro/2df. html.
} 
Table 1. Known spectroscopic members in the AAOmega field used as templates.

\begin{tabular}{|c|c|c|c|c|c|c|c|c|c|c|c|c|c|c|}
\hline $\begin{array}{c}\text { RA } \\
\text { h m s }\end{array}$ & $\begin{array}{l}\text { Dec } \\
\circ, \prime \prime\end{array}$ & $\begin{array}{c}J \\
\text { mag }\end{array}$ & \multicolumn{3}{|c|}{$\begin{array}{c}E W\left(\mathrm{H}_{\alpha}\right) \\
\AA\end{array}$} & \multicolumn{3}{|c|}{$\begin{array}{c}E W(\mathrm{NaI}) \\
\AA\end{array}$} & \multicolumn{3}{|c|}{$\begin{array}{c}E W(\mathrm{KI}) \\
\AA\end{array}$} & \multicolumn{2}{|c|}{$\begin{array}{ll}\text { SpT } & \text { new SpT }\end{array}$} & Name \\
\hline \multicolumn{2}{|c|}{ 16:10:50.00-22:12:51.8 } & 12.680 & 5.9 & -10.9 & -7.0 & 4.1 & 3.3 & 3.2 & 4.2 & 3.2 & 2.8 & 15.5 & $\bar{M}$ & DL \\
\hline \multicolumn{2}{|c|}{$16: 11: 17.11-22: 17: 17.5$} & 14.340 & & -3.6 & - & 2. & 3.4 & - & $\mathrm{N} / \mathrm{A}$ & N/A & - & .5 & M & $17^{2}$ \\
\hline \multicolumn{2}{|c|}{$16: 12: 37.58-23: 49: 23.4$} & 3.930 & -7.9 & -7.5 & -9.4 & .0 & 4.6 & 4.7 & 3.8 & 3.7 & 3.6 & 6.0 & M5.5 & $\mathrm{SCH} 16$ \\
\hline \multicolumn{2}{|c|}{$16: 12: 46.92-23: 38: 40.9$} & 13.650 & -15.2 & -13.6 & -8.4 & 3.9 & 4.3 & 4.0 & 5.4 & $\mathrm{~N} / \mathrm{A}$ & 3.4 & M6.0 & M6.25 & $\mathrm{SCH} 1$ \\
\hline \multicolumn{2}{|c|}{$16: 13: 12.12-23: 05: 03.3$} & 14.050 & -5.7 & -11.6 & -1.6 & 3.6 & 4.4 & 3.6 & 3.9 & 5.3 & 4.8 & M6.5 & M6.5 & SCH1613-2305 ${ }^{2}$ \\
\hline
\end{tabular}

References. (1) Martín et al. (2004); (2) Slesnick et al. (2006).

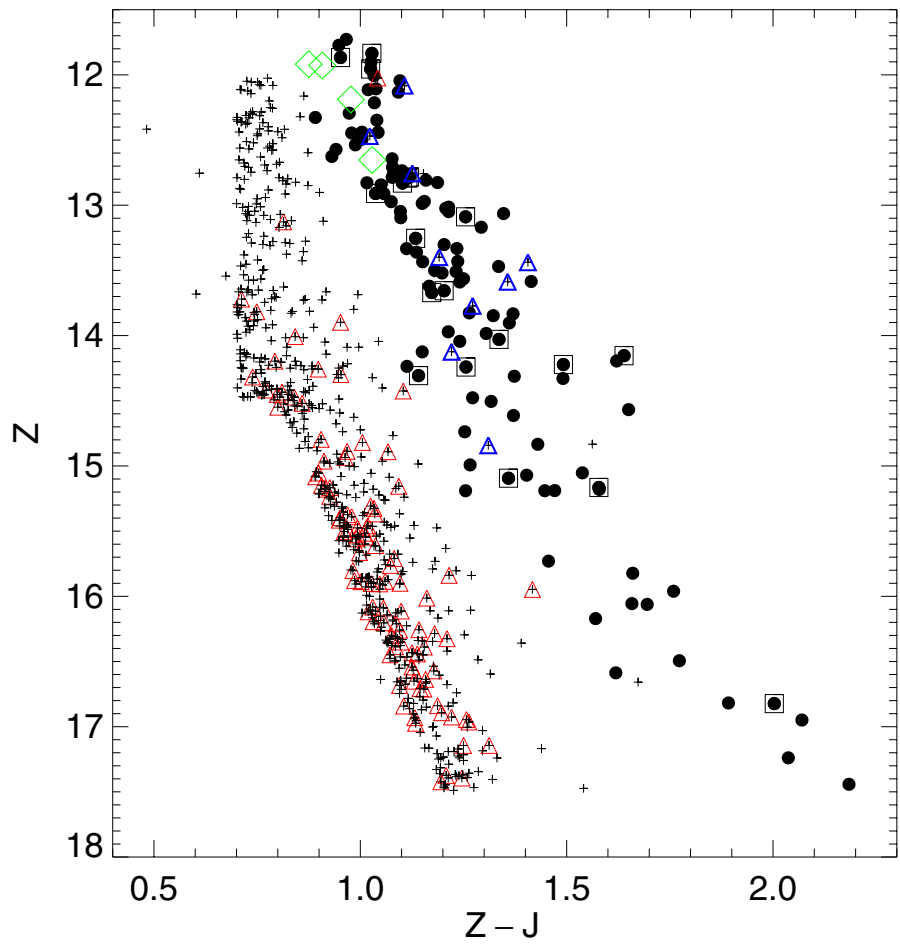

Fig. 1. $(Z-J, Z)$ colour-magnitude diagram for all objects in the AAOmega field-of-view (crosses) along with the new USco candidate members presented in this paper (filled dots) and photometric candidates lacking spectroscopy (open squares around filled dots). Overplotted are the nine objects classified as proper motion nonmembers by Lodieu et al. (2007b) and reclassified as spectroscopic members here (blue open triangles). Open red triangles represent a sample of stars in the AAOmega field classified as photometric non members but with proper motions and equivalent width measurements consistent with young objects. Open green diamonds represent the four photometric candidates rejected as spectroscopic members after our AAOmega follow-up.

also at a resolution of $R \sim 1350$, were simultaneously obtained on the blue arm where we employed the $580 \mathrm{~V}$ grating.

The observations were conducted during the nights 22-24 May 2007 by one of us (PDD). Seeing and transparency were decidedly variable throughout this period. The first night was dogged by substantial amounts of cloud and poor seeing (2.5-3.0 arcsec). The next night was largely clear but seeing was again poor (2.5-3.0 arcsec). On the final night the sky was clear and the seeing was substantially better $(0.8-1.5 \operatorname{arcsec})$. As this project was awarded an allocation of bright time, the moon was above the horizon for much of this period.

The observations were made with four fibre configurations at two positions on the sky: the first field was centered approximately at $(\mathrm{RA}, \mathrm{Dec}) \sim\left(16^{\mathrm{h}} 10^{\mathrm{m}},-23^{\circ}\right)$ whereas the second was positioned at $(\mathrm{RA}, \mathrm{Dec}) \sim\left(16^{\mathrm{h}} 14^{\mathrm{m}},-23^{\circ} 10^{\prime}\right)$. Each field had a set-up for a sample of bright $(Z=11.5-14.5 \mathrm{mag})$ and faint $(Z=14.5-17.5 \mathrm{mag})$ targets. Each field included science targets i.e. USco photometric candidates, USco known members confirmed spectroscopically by earlier studies, a sample of photometric non-members (or stars in the AAOmega field) pickedup randomly in the $(Z-J, Z)$ colour-magnitude diagram as well as "sky" fibres (typically 50 per field). The UKIDSS images were checked to confirm that no sources was detected at the sky positions down to the detection limit of the GCS $(J \sim 19.6$ mag $)$.

Single on-source integrations were set to $900 \mathrm{~s}$ and $1800 \mathrm{~s}$ for the bright and faint samples, respectively. Total on-source integrations typically range from $75 \mathrm{~min}$ to $345 \mathrm{~min}$ for the bright sample and from $270 \mathrm{~min}$ to $720 \mathrm{~min}$ for the faint sample. The total exposure time varies from target to target as $\sim 20$ objects have only one spectrum and the others have two or more spectra (up to four). The data were reduced in the standard manner using the 2DFDR package provided by the Australian Astronomical Observatory. We have separated the various files (bias, flats, and objects) into directories and performed an automated extraction of the one-dimensional spectra. More details on the workings of the 2DFDR package are discussed in Reid \& Parker (2010). We have measured the pseudo equivalent widths of the $\mathrm{H} \alpha$ emission line and the gravity-sensitive features in each spectrum to look for variability. In summary, we present the combined spectra for 94 USco photometric and proper motion candidates, five previously known members, 624 photometric non-members, and allocated 225 fibres to a sky position.

\subsection{Spectral variability}

We observed some level of variability between spectra of the same source. Thirteen objects out 94 candidates identified in the GCS show one or two discrepant spectra. Similarly, we detect variability in SCH162-2338 among the five previously known members. These variations may be caused by several effects: inhomogeneity in the fibre response, chromatic variation in optical distortion, or intrinsic variability in the atmospheric properties of low-mass stars and brown dwarfs. We have obtained a total of 233 spectra for the $94+5=99$ sources and found that $13+1=14$ objects are affected by this spectral variability, implying a rate below $15 \%$. Notably, most of the spectra affected show a significant level of fringing beyond $7500 \AA$. The final spectral classification should not be affected by more than half a subclass because each object has generally two identical spectra and one or two discrepant. The discrepant datasets have been removed for the subsequent analysis. 


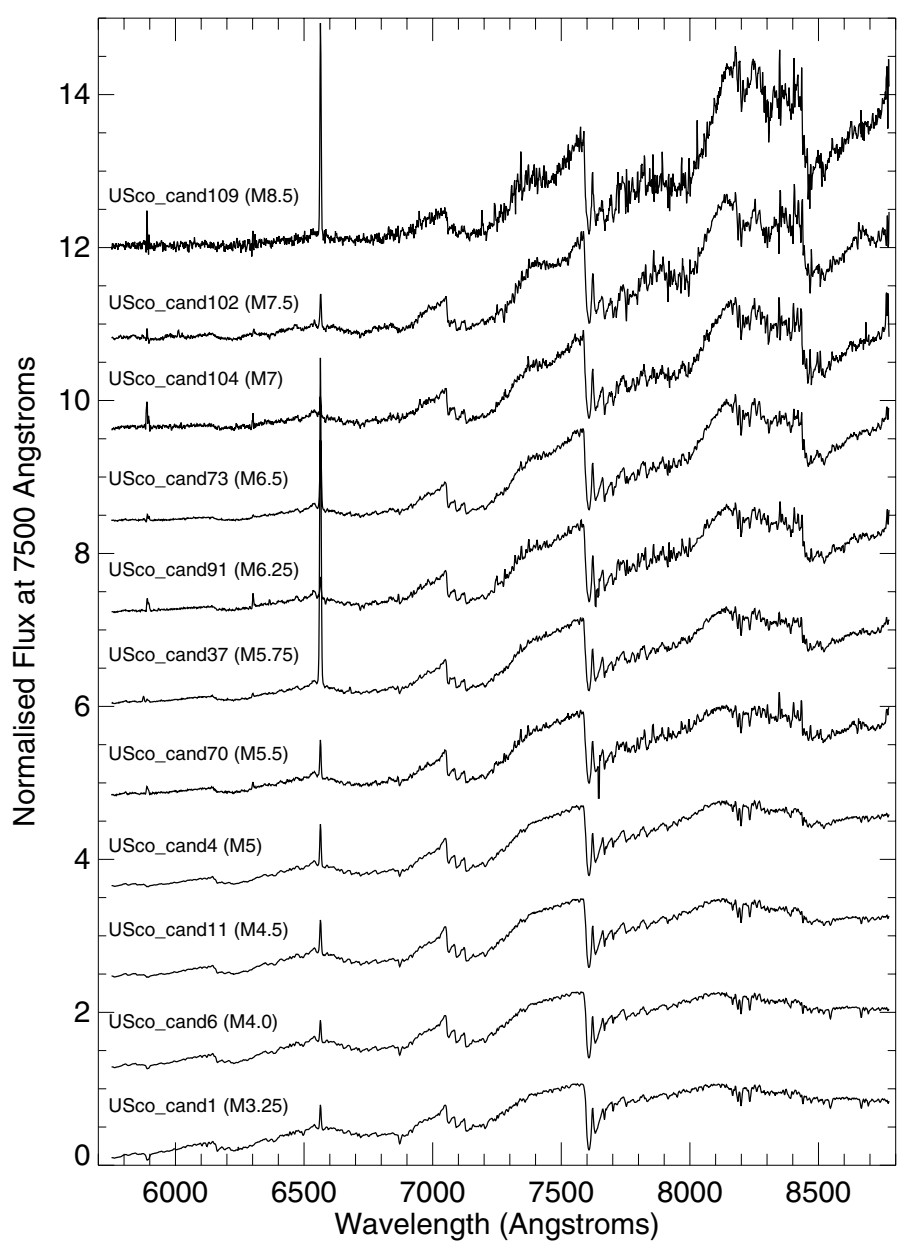

Fig. 2. Combined optical spectra of USco members obtained with AAT/AAOmega. The full range of spectral types (M3.25-M8.5) probed by our spectroscopic follow-up is shown with increasing spectral types from top to bottom. Spectra are shifted along the $y$-axis by increments of 1.2 for clarity.

\section{Membership assessment}

\subsection{Spectral types}

Among our targets, we have included five previously known spectroscopically confirmed members with spectral types ranging from M5.5 to M7.5 (Table 1, Martín et al. 2004; Slesnick et al. 2006). These sources represent our "primary" templates as they were observed with the same instrumental set-up as our photometric candidates. The direct comparison of the spectra of these five members suggests that SCH1611-2217, SCH1612-2338, and SCH1613-2305 have similar spectral types and are cooler than DENIS1610-2212 and SCH1612-2349 whose spectra look very much alike (Table 1).

In order to check their spectral types using the AAOmega spectra and to put our classification on an absolute scale, we have compared these five sources to young low-mass stars and brown dwarfs identified in the Chamaeleon I (M3-M9, Luhman 2004a, 2007), $\eta$ Chamaeleon (M3-M5.75, Luhman \& Steeghs 2004) and Taurus (M5.25-M9.5, Briceño et al. 1998; Luhman et al. 2003a) star-forming regions, and the Oph1622-2405 binary (M7.25 and M8.75 Luhman et al. 2007). The grid of spectral types available is fairly complete from M3 to M9.5 with a template every 0.25 subclass as follows: M3, M3.25, M4, M4.5, M4.75, M5, M5.25, M5.5, M5.75, M6, M6.25, M6.5, M7.25,
M7.5, M8.25, M8.5, M8.75, and M9.54. We have revised the spectral types of the five USco members as indicated in Table 1 by comparing them directly to the templates aforementioned. Uncertainties on the spectral types are typically given by the spacing of the grid i.e. $0.25-0.5$ subclass in most cases. The new spectral types match the previous estimates for known USco members within the uncertainties quoted by the earlier studies, except for SCH1611-2217 for which we derived M6.5 instead of M7.5 (Slesnick et al. 2006). We favoured this direct comparison over the determination of spectral types using spectral indices defined in the literature for field stars (e.g. the PC3 index, Martín et al. 1996) because they may not be reliable for young low-mass stars and brown dwarfs.

Among our sample of 94 GCS candidates, we found two objects of spectral types earlier than M, arguing that they are spectroscopic non members, UGCS J161315.65-232744.2 and UGCS J161209.48-223957.1 (Table 2). To assign spectral types to the remaining 92 candidates, we compared their combined spectra to our spectral templates: SCH1612-2305 (M6.5, Slesnick et al. 2006) and DENIS1610-2212 (M5.5, Martín et al. 2004). Then, we have compared the optical spectra to each other to create groups following a spectral type sequence. We have assigned a spectral type per group using young templates in Chamaeleon, Taurus, and Ophiuchus. We have found two sources with M2 spectral type which appear too faint for their spectral types. For this reason we consider them as non members in the rest of the paper. The spectral types span the M3.25-M8.5 range and are listed in Table A.1.

Columns 1 and 2 of Table A.1 provides the coordinates (J2000) and $J$-band magnitudes of 90 new spectroscopic candidate members of the USco association. Columns 3-6, 7-10, and 11-14 give the measurements (up to four) of the pseudoequivalent widths (in $\AA$ ) of the $\mathrm{H} \alpha$ line as well as the $\mathrm{NaI}$ and KI doublets, respectively, discussed in the following sections. We emphasise here that membership of the USco association is assigned based on the various criteria available to us: photometry, proper motion, $\mathrm{H} \alpha$ emission, gravity-sensitive features, lithium and magnesium lines when "available". The presence of contaminants cannot be completely ruled out but their level should be extremely low when using a combination of all these criteria. A long dash line indicates that either no spectrum is available or the signal-to-noise ratio is too low to publish a reliable measurement. Columns 15-18 list spectral indices; Cols. $19+20$ provide spectral types derived from the spectral indices and estimated from the spectral templates, respectively. Spectral types are accurate to 0.25 class for those earlier than M7.5 and half a subclass for later objects. Columns 21-23 provide the estimated effective temperatures (in $\mathrm{K}$ ), luminosities $\left(\log \left(L_{\mathrm{bol}} / L_{\odot}\right)\right)$, and masses (in $\left.M_{\odot}\right)$.

\subsection{Chromospheric activity}

In this section, we measure the equivalent widths of the $\mathrm{H} \alpha$ line at $6563 \AA$ for the 90 candidates whose optical spectra substantiate our claims about their membership of the USco association. The detection of strong $\mathrm{H} \alpha$ in emission in the optical spectral of young low-mass stars and brown dwarfs is generally assumed to indicate youth. We used the SPLOT task under IRAF ${ }^{5}$ to measure pseudo-equivalent widths to an accuracy of $1 \AA$ or

\footnotetext{
4 Spectra kindly provided by Dr. Kevin Luhman.

5 IRAF is distributed by National Optical Astronomy Observatory, which is operated by the Association of Universities for Research in Astronomy, Inc., under contract with the National Science Foundation.
} 
N. Lodieu et al.: Multi-fibre optical spectroscopy of low-mass stars and brown dwarfs in Upper Scorpius

Table 2. Summary details of spectroscopic non-members, including coordinates, photometry, proper motions, and approximate spectral types.

\begin{tabular}{ccccccccccc}
\hline \hline $\begin{array}{c}\text { RA } \\
\text { h s m }\end{array}$ & $\begin{array}{c}\text { Dec } \\
{ }^{\prime}{ }^{\prime \prime}\end{array}$ & $\begin{array}{c}Z \\
\mathrm{mag}\end{array}$ & $\begin{array}{c}Y \\
\mathrm{mag}\end{array}$ & $\begin{array}{c}J \\
\mathrm{mag}\end{array}$ & $\begin{array}{c}H \\
\mathrm{mag}\end{array}$ & $\begin{array}{c}K \\
\mathrm{mag}\end{array}$ & $\begin{array}{c}\mu_{\alpha} \cos \delta \\
\mathrm{mas} / \mathrm{yr}\end{array}$ & $\begin{array}{c}\mu \delta \\
\mathrm{mas} / \mathrm{yr}\end{array}$ & Type & Name \\
\hline 16:13:15.65 & $-23: 27: 44.2$ & 11.918 & 11.548 & 11.043 & 10.279 & 10.053 & -7.3 & -8.7 & $\mathrm{dK}$ & cand8 \\
16:12:09.48 & $-22: 39: 57.1$ & 12.189 & 11.764 & 11.212 & 10.425 & 10.149 & 10.1 & -7.8 & $\mathrm{dK}$ & cand13 \\
\hline 16:13:20.53 & $-22: 29: 16.0$ & 11.929 & 11.531 & 11.021 & 10.578 & 10.162 & -17.2 & -11.1 & $\mathrm{M} 2$ & cand7 \\
16:07:08.81 & $-23: 39: 59.9$ & 12.653 & 12.127 & 11.624 & 11.044 & 10.714 & -4.7 & -14.5 & $\mathrm{M} 2$ & cand23 \\
\hline
\end{tabular}

better (depending on the strength of the line). M dwarfs are longlived and remain active for a long time. A large fraction of M3M9 field dwarfs are active, on average $40 \%$ but with a range from $20 \%$ for M3 up to $\sim 100 \%$ for the latest M dwarfs (West et al. 2008). However, only $\sim 15 \%$ of old field $\mathrm{M}$ dwarfs exhibit strong $\mathrm{H} \alpha$ emission with equivalent widths less than $-5 \AA$.

We detect $\mathrm{H} \alpha$ in emission in the optical spectra of the USco candidate members (Fig. 3), except one source for which $\mathrm{H} \alpha$ is in absorption despite its late spectral type, UGCS J161340.79-221946.1 (M7.5). Absorption lines occur for stars in standard evolutionary states such main-sequence or post-main sequence but are unlikely in the pre-main sequence stage. However, due to the large amount of fringing and the low signal-to-noise of this particular spectrum, we prefer to keep it as a possible candidate. We also observe different levels of variability in our equivalent width measurements reported in Table A.1. As an example, we have three sources, UGCS J161216.09-234425.0, UGCS J161126.30-234006.1, and UGCS J161047.13-223949.4, where we detect H $\alpha$ in emission in only two of the four spectra. In particular, the latter shows strong $\mathrm{H} \alpha$ emission with equivalent widths of $\sim-55$ and $\sim-30 \AA$ while no emission is detected in the other two spectra. Nonetheless, we keep these sources as members because their spectra are otherwise consistent with young low-mass stars. These measurements show that variability is common among young low-mass stars and brown dwarfs and that the absence of $\mathrm{H} \alpha$ alone is not sufficient to rule out youth as a characteristic of low-mass stellar and substellar objects (Martin et al. 2010).

The $\mathrm{H} \alpha$ emission can be due to, on the one hand, chromospheric activity, and, on the other hand, to accretion or strong winds. The latter is usually inferred from the strong levels of $\mathrm{H} \alpha$ emission. To quantify this statement, Barrado y Navascués \& Martín (2003) have defined an empirical boundary between non-accreting and accreting M-type stars and brown dwarfs. We have identified 11 new accreting candidates that lie significantly above this empirical boundary (dotted line in Fig. 3). One source, UGCS J160648.18-223040.1, lies outside the limits of the plot displayed in Fig. 3 because of its extremely strong $\mathrm{H} \alpha$ emission. In the first spectrum, we have measured an equivalent width $(E W)$ of $-1780 \AA$ whereas the emission is weaker $(E W=-730 \AA)$ in the second spectrum although as strong as the strongest $\mathrm{H} \alpha$ emission detected in a member of the young $\sigma$ Orionis cluster (SOri 71, $E W=-705 \AA$ Barrado y Navascués et al. 2002). We have identified another object, UGCS J160723.82-221102.0 which also exhibits strong $\mathrm{H} \alpha$ emission with equivalent width measurements of -671.0 and $-431.5 \AA$, comparable to SOri 71. Moreover, we have measured $\mathrm{H} \alpha$ equivalent widths of order -155 to $-135 \AA$ for USco J161354.34-232034.4.

\subsection{Surface gravity}

To further assess the membership of our USco photometric candidates, we have measured the pseudo-equivalent widths of the

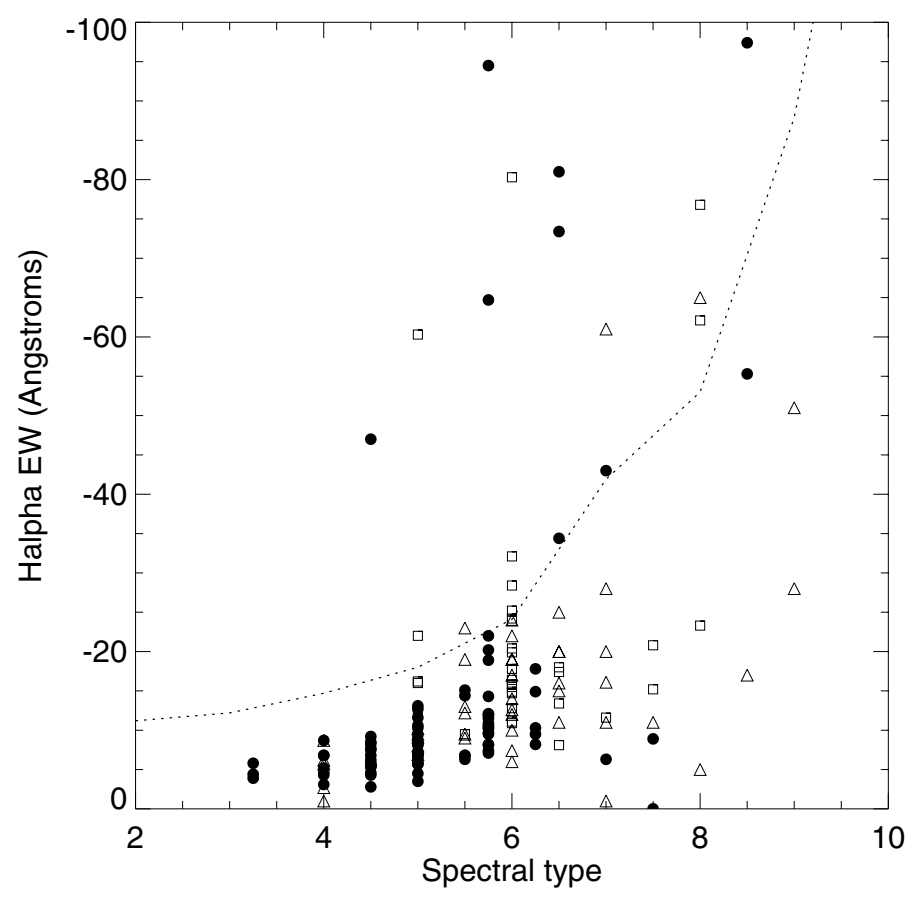

Fig. 3. H $\alpha$ equivalent widths ( $E W \mathrm{~s}$; in $\AA$ ) as a function of spectral type ( $4 \equiv \mathrm{M} 4 ; 5 \equiv \mathrm{M} 5$; etc.) for all photometric candidates confirmed as spectroscopic members (filled dots) in this study. Three objects lie outside this plot due to their extremely strong $\mathrm{H} \alpha$ emission. We have added data points from earlier studies for comparison: open triangles come from Ardila et al. (2000) and Martín et al. (2004) and open squares are from Slesnick et al. (2006).

gravity-sensitive Na I (8183/8195 $\AA$ ) and K I (7665/7699 $\AA)$ doublets in each individual spectrum (Table A.1).

The left-hand side panel of Fig. 4 shows the behaviour of the equivalent widths of the $\mathrm{Na}$ I doublet as a function of spectral type. Its equivalent width appears constant as a function of spectral type with a mean value of $3.5-4 \AA$ and a dispersion of $1 \AA$. The upper envelope for Upper Sco candidate members is around 5.5 $\AA$ as seen to Fig. 4 in Martín et al. (2004), consistent with the trend of our measurements (we find an upper limit of $5 \AA$ ). The trends in open clusters like the Pleiades (Martín et al. 1996) and Alpha Per (Lodieu et al. 2005a) indicate mean values of $5 \pm 2 \AA$ for the equivalent widths of the Na I doublet in the M4-M8 spectral type range whereas old field dwarfs have equivalent widths typically larger than $6 \AA$ (Martín et al. 1996). Therefore, our measurements are consistent with general trends and consolidate our conclusion that our USco photometric candidates are young objects.

The right-hand side panel of Fig. 4 shows the behaviour of the K I doublet as a function of spectral type for the new USco candidate members. The equivalent widths of the doublet exhibits an increase as a function of spectral type and values in the 2-6 Å range. 

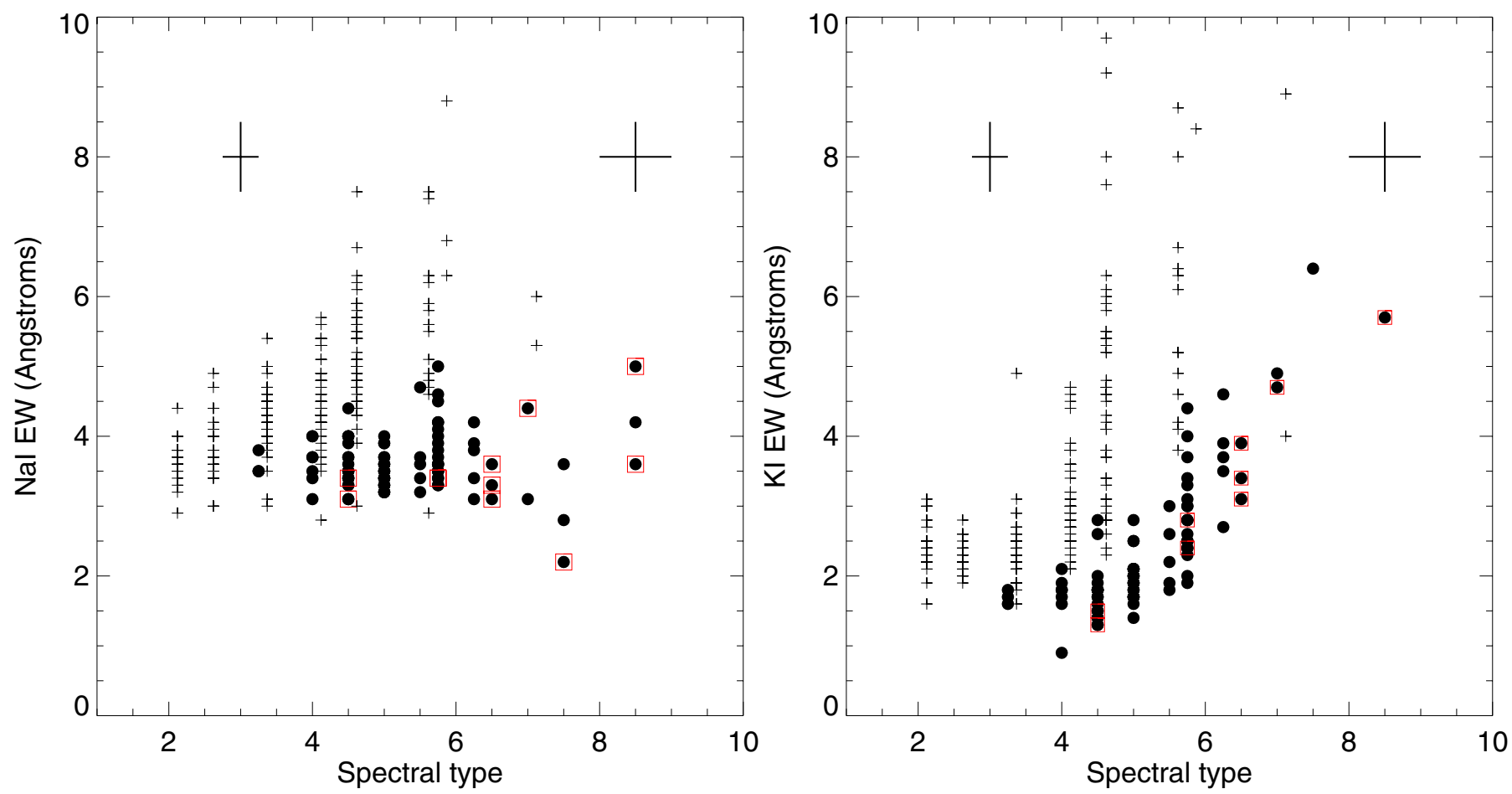

Fig. 4. Na I (left) and K I (right) equivalent width measurements (in $\AA$ ) as a function of spectral type ( $4 \equiv$ M4; $5 \equiv$ M5; etc.) for all the photometric candidates confirmed as spectroscopic members. Typical error bars on the equivalent width measurements are marked. Some of the latest type members lack measurements for the KI doublet because of the nearby strong oxygen telluric band. The candidates for accretion i.e. which exhibit $\mathrm{H} \alpha$ equivalent widths larger than the empirical boundary defined by Barrado y Navascués \& Martín (2003) are highlighted with red squares. The stars lying in the AAOmega field have been added as crosses and shifted in spectral types by +0.12 for clarity.

On both panels of Fig. 4, we have overplotted the equivalent width measurements of 180 stars lying in the AAOmega fieldof-view and classified as $\mathrm{M}$ dwarfs without $\mathrm{H} \alpha$ in emission (red crosses). The mean values of the equivalent widths of the Na I and $\mathrm{KI}$ doublets for these field $\mathrm{M}$ dwarfs is clearly higher than the average of the new USco candidate members for each spectral type. One hundred and twenty seven $(\sim 70 \%)$ stars in the AAOmega field have Na I doublet equivalent widths larger than $4 \AA$, pointing towards an older age on average at a given spectral type than for our USco candidate members. The difference is even more marked for the K I doublet.

We have also computed the temperature (TiO- $\lambda 7140$ and TiO- $\lambda 8465)$ and gravity (Na-8189) sensitive indices designed by Slesnick et al. (2006) for the new USco candidates (filled circles) and previously known members (open squares in Fig. 5). Figure 5 shows the same plots with the same $(x, y)$ scales as in Fig. 2 of Slesnick et al. (2006) and Figs. 10, 11 of Slesnick et al. (2008) for direct comparison between their samples and ours. Note that the six faintest sources of our sample of candidate members are not included in Fig. 5 because of strong fringing beyond $8000 \AA$ affecting the computation of the TiO- $\lambda 8465$ and $\mathrm{Na}-8189$ indices. Our values of the three indices agree within $10 \%$ with their values published in their Table 1 . The trend in the left-hand side plot of Fig. 5 confirms the range in spectral types (M3.25-M8) derived from direct comparison with young templates. A few objects lie below the sequence, a position attributed to the occurence of veiling by Slesnick et al. (2006). Similarly, the mean values of the Na-8189 for our sample is around 0.9 with a dispersion of 0.3 , consistent with the range of values $(\sim 0.86-0.96)$ reported by Slesnick et al. (2006) and Slesnick et al. (2008).
The positions of the stars in the AAOmega field-of-view in the left-hand side diagram of Fig. 5 follow the general trend of the USco candidates mainly because this diagram is independent of the age (i.e. gravity). However, the locations of these objects in the right-hand side diagram differ from the positions of the USco candidates and appear intermediate between field dwarfs and pre-main-sequence stars (blue and green crosses in Fig. 2 of Slesnick et al. (2008), respectively). This trend points towards an intermediate age for the stars in the AAOmega field and corroborates the conclusions drawn from the equivalent widths of the Na I doublet. Their position in the $(Z-J$, $Z$ ) colour-magnitude diagram displayed in Fig. 1 indicates that these objects are, on average, fainter than the USco members for the same spectral type, suggesting that they are located beyond the USco association.

\subsection{Other features: lithium and magnesium}

Although searching for the presence or absence of the lithium absorption line at $6707.8 \AA$ was not originally an aim of this project, we are able to detect that line thanks to a combination of the moderate resolution $(R \sim 1100)$ and the high quality of the spectra obtained for the brightest sources $(J \leq 13 \mathrm{mag}$ and $Z \leq 14.5 \mathrm{mag}$ ). The strength of the equivalent width varies from object to object and is of the order of $0.2-0.5 \AA$. The lithium is detected in absorption in almost all photometric candidates confirmed spectroscopically on the basis of the presence of $\mathrm{H} \alpha$ and weak gravity features (top panel in Fig. 6), again adding credence to their inferred youth and membership of the USco association. 
N. Lodieu et al.: Multi-fibre optical spectroscopy of low-mass stars and brown dwarfs in Upper Scorpius
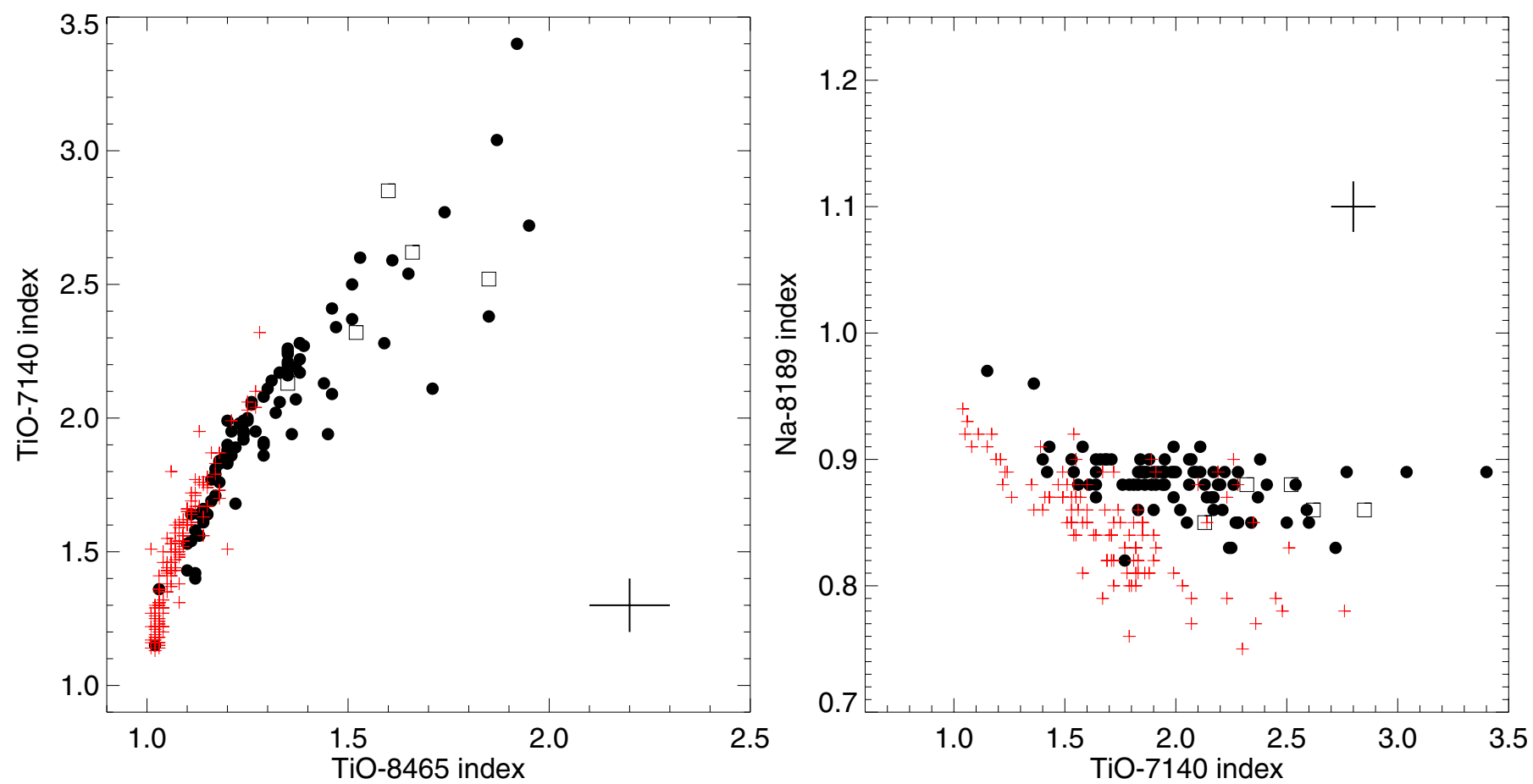

Fig. 5. Temperature (left) and gravity (right) sensitive indices for the new USco candidate members (filled circles), previously known members (open squares), and stars in the AAOmega field-of-view (crosses). Typical error bars are shown. Plots originally proposed by Slesnick et al. (2006).
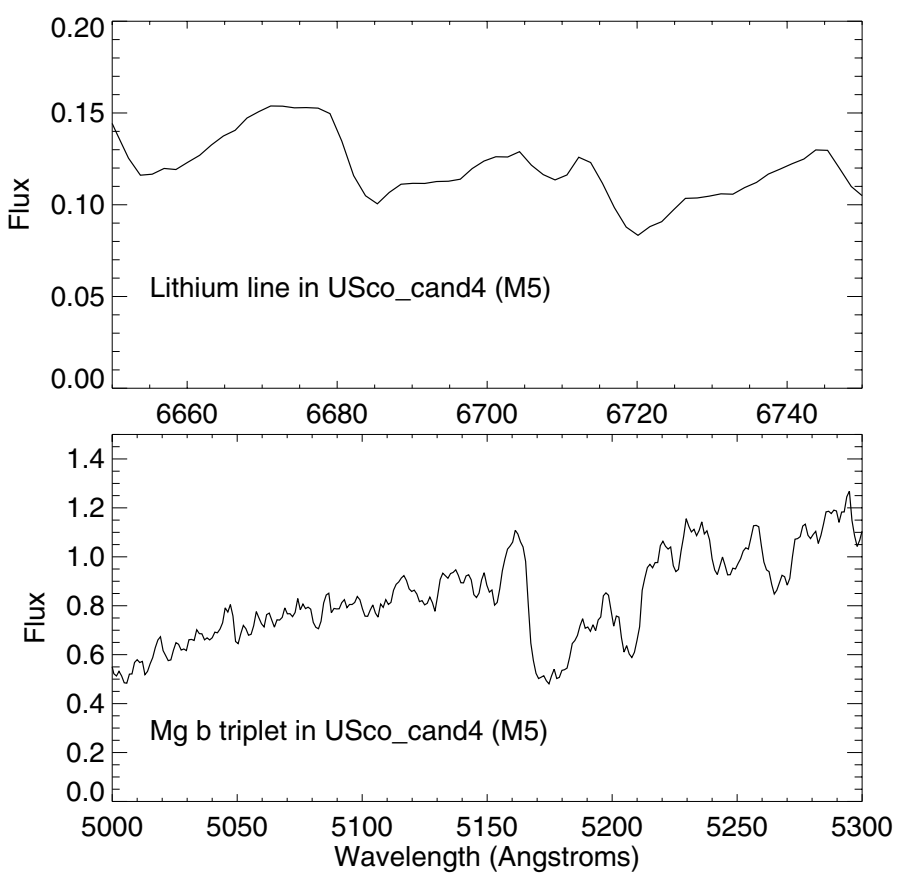

Fig. 6. Region around the magnesium triplet at $\sim 5200 \AA$ (bottom) and the lithium line at $6708.8 \AA$ for one USco members (USco_cand4; M5).

In the blue part of the spectra, we detect the presence of the $\mathrm{Mg} \mathrm{b}$ triplet around $5200 \AA$ with an excellent signal-to-noise for the brightest USco members (bottom panel in Fig. 6). The full spectral range provided by the blue grating (3740-5720 $)$ at a resolution of $R \sim 1350$ could be used to study the accretion rates in a large sample of young low-mass stars and brown dwarfs at 5 Myr (e.g. Herczeg et al. 2009) but such a study is beyond the scope of this paper.

\subsection{Level of contamination}

Among the 94 photometric and proper motion candidates followed-up spectroscopically with AAOmega, we have confirmed the membership of 90 translating into a success rate of the original selection of $\sim 96 \%$. Two contaminants listed in Table 2 appear to be background stars and the other two field early-M dwarfs. This result demonstrates the performance of the photometric and astrometric selections described in Lodieu et al. (2006) and Lodieu et al. (2007b).

We note that four objects were previously confirmed through their near-infrared cross-dispersed spectra (Lodieu et al. 2008). The spectral types of USco J160830.49233511.0 (M8.5 in the optical vs. M9 in the nearinfrared), USco J160648.18-223040.1 (M8.5 vs. M8), and USco J161047.13-223949.4 (M8.5 vs. M9) agree within a subclass. The optical and near-infrared spectral types of USco J160723.82-221102.0 differ by several subclasses (M7.5 vs. L1) as noted by Herczeg et al. (2009) who independently classified it as a M8.5 dwarf. Another object, USco J161421.44-233914.8, rejected by our near-infrared spectroscopic follow-up, is now reclassified as a member with a M7 spectral type according to the AAOmega spectrum. We have checked the input coordinates and positions in both runs and cannot find out the reason for this discrepancy. Unfortunately, we could not reach fainter candidates to confirm our nearinfrared classification with optical spectroscopy. The former has been criticised by Herczeg et al. (2009) on the basis of only two sources where the classifications are discrepant. We should point out that younger $\mathrm{M}$ dwarfs are hotter than their field counterparts, implying that the vanadium oxide (VO) band should remain at later spectral types for younger ages and thus lead an earlier spectral classification (Martín et al. 1996).

For the faintest USco candidates with estimated masses below $0.03 M_{\odot}$ according to theoretical models, our earlier study showed that 19 out of 20 photometric candidates are indeed 
spectroscopic members (Lodieu et al. 2008). Their near-infrared cross-dispersed spectra confirm them as ultracool dwarfs with features characteristics of young objects and effective temperatures in the $2700-1800 \mathrm{~K}$ range. Therefore, the level of contamination in the $0.03-0.006 M_{\odot}$ mass range is of the order of 5\% after reclassifying USco J161421.44-233914.8. Over the full magnitude and mass ranges probed in USco by the GCS, our success rate is $\sim 95 \%$.

\section{Probing the efficiency of the original selection}

In addition to the USco members discussed in Sect. 4.1, we assigned 624 fibres to sources located to the blue side of the lines used for our photometric selection objects referred as to "stars in the AAOmega field"). Among these extra sources, we have identified a large number of stars (Sect. 5.1), proper motion non members, as well as three accreting sources (Sect. 5.4). We have used this large sample to estimate the efficiency of the original photometric (Sect. 5.3) and proper motion (Sect. 5.2) selections described in Lodieu et al. (2007b) based on multiple criteria, including the magnitudes, spectral types, strengths of the equivalent width of activity and gravity features.

\subsection{The sample of stars in the AAOmega field}

We have assigned fibres to over 600 stars or point sources not selected by our photometric study as members of the USco association (crosses in Fig. 1, Lodieu et al. 2007b). We provide the coordinates, photometry, astrometry and tentative spectral types for all stars in the AAOmega field (Table B.1) as well as the optical spectra to the community ${ }^{6}$ as they may be useful to future surveys of the region or to other type of scientific exploitation. The large majority of these objects do not belong to the Upper Sco association, except the ones discussed in the following sections and listed in Table C.1.

We have assigned rough spectral types accurate to a few spectral classes using a combination of criteria including direct comparison with low-resolution spectra downloaded from the European Southern Observatory database ${ }^{7}$ and expected strengths of major lines and absorption bands (e.g. NaD, TiO, $\mathrm{CaH}, \mathrm{Ca}$ I, Na I) as a function of the luminosity class and spectral types (Table 5 of Kirkpatrick et al. 1991; and spectra in Turnshek et al. 1985).

The full sample of spectra can be divided up into several groups for which we have chosen one template because of the high quality of its spectrum. The majority of objects enters in the class of $M$ dwarfs with spectral types later than M2 (right panel of Fig. B.2). We have also identified a large number of late$\mathrm{K} /$ early-M dwarfs (K7-M1) with different levels of reddening and late-G giants (left-hand side of Fig. B.2). Finally, we have classified three sources as young active flare M dwarfs (details in Sect. 5.4), two white dwarfs, 18 objects that we were unable to classify due to problems with the fibre response or low signal-tonoise, and 11 sources have no signal. Tables, plots and selected templates are detailed in Appendix B.

\footnotetext{
6 Tables and AAOmega optical spectra taken with the blue (3740-5720 ̊) and red (5600-8800 ̊) gratings are available at WWW . iac . es/galeria/nlodieu/publications/.

7 www.eso.org/instruments/isaac/tools/lib/index.html
}

\subsection{Contamination in the proper motion selection}

In Lodieu et al. (2007b), we used a $2 \sigma$ clip to reject proper motion non members, implying that our selection should have included $\sim 95 \%$ of members but missed $\sim 5 \%$ of true members (assuming normally distributed errors). In this section, we aim to address the question related to the level of contamination present in our original cut in the proper motion selection: do we find that $5 \%$ of the proper motion non members listed in Table $\mathrm{C} 1$ of Lodieu et al. (2007b) are actually members?

In Lodieu et al. (2007b), we discarded 23 photometric candidates as proper motion non members ${ }^{8}$. Ten of these 23 proper motion non members were included in our spectroscopic followup having been assigned a fibre (Table C.1). We have looked at their spectral types, $\mathrm{H} \alpha$ and $\mathrm{Na}$ I equivalent widths, and the presence of lithium to investigate their membership. Among these 10 sources, only one is rejected as a member based on its high $\mathrm{Na}$ I equivalent width. The remaining nine objects show $\mathrm{H} \alpha$ in emission and weak gravity-sensitive features, and have spectral types between M4 and M6, consistent with young objects. Among those nine sources, six have a clear lithium detection while the other three may exhibit lithium in absorption but the detection is only tentative.

The results, detailed above, are summarised in Table C.1. Column 1 of Table C. 1 gives the name of the object. Column 2 provides the $Z$-band magnitude; Cols. 3 and 4 the proper motion (mas/yr) in right ascension and declination, respectively; Col. 5 the spectral types; Cols. 6 and $7 \mathrm{H} \alpha$ and $\mathrm{Na}$ I equivalent widths ( $E W \mathrm{~s}$ in $\AA$ ), both individual measurements and the sum; Col. 8 the presence of lithium (Y $\equiv$ Yes); Col. 9 a note on the proper motion; Col. 10 the final decision on the membership, and the last column the proper motions from UCAC3 (Zacharias et al. 2010).

We have checked the UCAC3 catalogue for the other 13 sources classified as proper motion non members in Table C1 of Lodieu et al. (2007b). Seven objects brighter than $Z \leq$ 13 mag have a UCAC3 counterpart and astrometry accurate to a few mas/yr (Zacharias et al. 2010). The quality of the astrometry provided by UCAC3 supersedes our previous proper motion measurements and is useful to constrain membership in USco (Bouy \& Martín 2009). Three are clearly proper motion non members: USco J160937.85-212319.0, and the (possible) wide binary composed of USco J161002.67234439.5 and USco J161002.86-234440.9. Another object, USco J161626.20-235048.8, is possibly not a member. The other three bright objects are proper motion members but lack optical spectra because they were not assigned a fibre during our spectroscopic follow-up. The remaining six (out of 13) are not in the UCAC3 online catalog mainly because they are too faint.

We have made a comparison between the proper motions in right ascension and declination from UCAC3 and the ones derived from the 2MASS/GCS cross-match (Lodieu et al. 2007b). This sample of stars with $J=10.75-13$ mag contains 2815 sources. The agreement between both measurements is usually good with Gaussian equivalent root-mean-squares of 8.04 and $7.95 \mathrm{mas} / \mathrm{yr}$ in right ascension and declination, respectively. For the 43 USco candidates with UCAC3 measurements, we find median absolute deviations of 11.1 and 6.53 mas/yr in right ascension and declination, respectively. These error bars estimated from both datasets appear very similar in size.

\footnotetext{
83 sources in Table C1 of Lodieu et al. (2007b) are common to the sample of photometric non members listed in their Table B1.
} 
To summarise, we find that our $2 \sigma$ clip in the proper motion mis-classified 12 members (out of 17) as proper motion non members (and possibly up to 18 out of 23). In Lodieu et al. (2007b) we rejected 23 out of 139 photometric candidates as proper motion non members because they lay beyond the $2 \sigma$ cut off. For normally distributed errors, we would expect $5 \%$ of $139=7$ photometric members to be outside $2 \sigma$ just by chance. Here we find that between 12 and 18 objects are actually members i.e. a factor of two larger than the nomical 5\%. Only one object, USco J161412.41-221913.3, does lie outside the $3 \sigma$ circle centered on the cluster mean proper motion (Table C.1), suggesting that our original selection was pragmatic and threw away some bona-fide members. Future astrometric selection should rather employ $3 \sigma$ clips to optimize the selection of members. The uncertainties in the proper motion measurements from the 2MASS/GCS cross-match was typically $10 \mathrm{mas} / \mathrm{yr}$ down to $J=15.5 \mathrm{mag}$. We hope to improve the reliability of the astrometry by combining the UKIDSS GCS with the second epoch planned within the framework of the VISTA public surveys (Emerson 2001) ${ }^{9}$.

\subsection{Completeness of the selection}

In this section, we discuss the completeness of the original photometric selection designed to identify members in the USco association (Lodieu et al. 2007b). To test this original photometric selection, we have computed lower and upper limits for the completeness by comparing the number of photometric candidates followed-up spectroscopically to the 129 photometric candidates identified in the GCS after pure photometric selection (Lodieu et al. 2007b). A total of 94 sources have optical spectra and four are non members while 20 were observed in the near-infrared (one is classified as a non member). The remaining sources currently lack spectroscopic follow-up. Therefore, the lower limit for the efficiency of our selection is $\sim 81 \%(=(90+19-5) / 129)$ and the upper limit is $\sim 96 \%(=(90+19-5) /(94+20-5))$.

Secondly, we have investigated the completeness of the photometric selection by examinining the characteristics of stars lying within the AAOmega field-of-view to which a fibre was assigned. After keeping only stars whose proper motion is within $3 \sigma$ from the mean proper motion of the cluster $((-11$, -25) mas/yr, de Bruijne et al. 1997; Preibisch et al. 1998), we are left with 331 candidates. As a second step we have removed giants and late-K/early-M dwarfs as well as M dwarfs which do not exhibit $\mathrm{H} \alpha$ in emission, leaving a total of 107 candidates. Then, we have kept only sources with a $\mathrm{NaI}$ doublet equivalent width less than $5 \AA$, leaving 58 candidates. We note that 10 ( 9 are members but 8 satisfied the above criteria in the original selection) of these 58 sources lie to the red of the photometric selection and correspond to the proper motion candidates in Table C.1. To summarise, the completeness of our photometric selection is better than $129 /(129+58) \sim 69 \%$, with an upper limit of $129 /(129+8) \sim 94 \%$. These numbers are consistent with the values derived from our optical and near-infrared spectroscopic follow-up as described in the first paragraph of this section.

Table D.1 gives the coordinates (J2000), ZYJHK photometry, proper motions (mas/yr), equivalent widths of the $\mathrm{H} \alpha$ and Na I lines (in $\AA$ ), and spectral types for the 50 (58-8) candidates with proper motion within $3 \sigma$ from the cluster mean motion and with equivalent widths measurements of $\mathrm{H} \alpha$ and the $\mathrm{Na}$ I doublet consistent with membership. These objects are plotted as

${ }^{9}$ More details on VISTA at www.vista.ac.uk. red open triangles in the $(Z-J, Z)$ colour-magnitude diagram (Fig. 1).

Among the 58 sources left after the selection based on proper motions and the equivalent widths of the $\mathrm{H} \alpha$ line and the $\mathrm{Na}$ I doublet, 11 exhibit lithium in absorption and 39 show noticeable N II emission at $6583 \AA$. Of the 39 with N II emission, four exhibit strong S II emission lines at $6716 \AA$ and $6731 \AA$, indicative of a high excitation TTauri-like phase. In the full sample of stars in the AAOmega field-of-view, 87 sources show N II in emission and 16 out of 87 exhibit strong S II emission lines. They may belong to the nearby Upper Centaurus Lupus association as the difference in the age (13-16 Myr vs. $5 \mathrm{Myr}$ ) and the distance (145 pc vs. 160 pc) would result in members of Upper Centaurus Lupus being one magnitude fainter than their USco counterparts according to theoretical models (Baraffe et al. 1998). They could also have escaped from the nearby $\rho$ Oph star-forming region ( $\leq 1 \mathrm{Myr} ; 140 \mathrm{pc}$ ) where the occurence of disks is expected to be higher than in Upper Sco (Haisch et al. 2001).

\subsection{Three young accreting sources}

Among stars in the AAOmega field followed-up spectroscopically, we have detected three accreting sources with strong emission lines. These sources, UGCS J161450.31-233240.0 (M4.5, Fig. B.2), UGCS J161503.64-235417.7 (M4), and UGCS J160729.59-230822.4 (M3), exhibit strong emission lines of $\mathrm{H} \alpha$ and $\mathrm{He}$ I with pseudo equivalent widths of $(-108$, $-3.0),(-187,-2.2)$, and $(-150,-3.0) \AA$, respectively. We also clearly detect the sodium doublet at $5875 / 5890 \AA$, the calcium triplet at $8498 / 8542 / 8662 \AA$, and OI forbidden emission lines at $\sim 6300$ and 6363 , and $8446 \AA$ in all three sources. The Ca triplet is weaker in UGCS J161503.64-235417.7 than in the other two objects while the OI emission is comparable with pseudo equivalent widths between 3.6 and $5.3 \AA$. The proper motions of these three sources are given in Table B.1: only UGCS J161503.64-235417.7 has a proper motion inconsistent with the USco mean proper motion. We have added to Table D.1 the two accreting sources with proper motions consistent with USco for consistency but we do not consider them as members in the rest of the paper because they were originally classified as photometric non members.

The presence of a disk and strong emission lines indicates a young age and a high excitation phase like in T Tauri stars. The OI forbidden lines are indicative of outflows. These objects, however, did not fall in our initial photometric selection because of their colours. Indeed, young disk-bearing sources tends to exhibit unusual colours and magnitudes leading to their rejection as photometric candidates (e.g. Luhman et al. 2008; Lodieu et al. 2009; Mayne \& Harries 2010). A mid-infrared spectroscopic study of these two objects would shed light on the amount of dust re-processing (e.g. Riaz et al. 2009) and lead to better constraints on the age of these sources in order to find out whether they are members of USco or of a younger population. Additional study of the sources listed Table D.1 should be undertaken to find out if a pure photometric search is indeed biased towards sources with low accretion rates (Mayne \& Harries 2010).

\section{The spectroscopic mass function}

In this section, we consider all photometric candidates confirmed spectroscopically as members of USco. The original photometric sample contains 129 sources (Lodieu et al. 2007b). After the optical spectroscopic follow-up conducted with AAOmega 
Table 3. Number of sources as a function of spectral type along with the assigned effective temperatures (in K) and $J$-band bolometric corrections.

\begin{tabular}{|c|c|c|c|c|c|c|c|c|c|c|c|c|c|c|c|}
\hline M3.5 & M4.0 & M4.5 & M5.0 & M5.5 & M5.75 & M6.25 & M6.5 & M7.0 & M7.5 & M8.0 & M8.5 & M9.0 & L0.0 & L1.0 & L2.0 \\
\hline 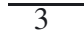 & $\sqrt{7}$ & 7 & 8 & 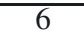 & 8 & $F$ & 2 & $\sqrt{n}$ & 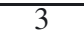 & $\overline{0}$ & 3 & 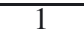 & 6 & 6 & 1 \\
\hline 340 & 3270 & 3200 & 312 & 3060 & 302 & 2935 & 2910 & 2880 & 2800 & 27 & 25 & 2400 & 2250 & 2100 & 1950 \\
\hline 1.85 & 1.86 & 1.86 & 1.90 & 2.02 & 2.03 & 2.04 & 2.04 & 2.04 & 2.02 & 2.01 & 2.00 & 1.97 & 1.92 & 1.92 & 1.91 \\
\hline
\end{tabular}

(90 members; this study) and the near-infrared cross-dispersed spectroscopy obtained for the faintest candidates (19 members; 5 in common with optical follow-up Lodieu et al. 2008), we have a final spectroscopic sample of 104 members. We should add to this sample the nine members originally rejected on astrometric grounds (Table C.1), yielding a total of 113 members in 6.5 square degrees near the centre of the USco association.

Spectroscopy is missing for 20 photometric candidates. We have looked at the histograms of the number of sources with and without spectroscopy as a function of magnitude, dividing up each sample into bins of $0.5 \mathrm{mag}$. The numbers of candidates without spectroscopy is of the order or below the square root of the number of objects with optical spectra in each magnitude bin. Therefore, we do not expect any artificial gap or peak in a statistical point-of-view due to the incomplete spectroscopic followup. However, this effect of incompleteness is hard to quantify until spectroscopy is obtained for all photometric candidates. Furthermore, most of the photometric candidates without spectroscopy are brighter than $J=14 \mathrm{mag}$, corresponding to spectral types of M6.5 or earlier i.e. where the bulk of spectroscopic members is located. Thus, the influence on the peak of the luminosity function should be minimal.

\subsection{Effective temperatures}

The spectral types of these members from the spectroscopic sample range from M3.25 to L2. The uncertainty is typically 0.25 subclass for spectral types earlier than M7.5 and 0.5 subclass for later types.

To estimate the temperature associated with each spectral type, we have used the temperature scale defined by Luhman (1999) for young objects with spectral types between M3 and M9 (Table 3). This scale uses temperatures intermediate between field dwarfs and giants and is valid up to M9. Typical uncertainties on each individual temperature are of the order $50 \mathrm{~K}$. More recently, Rice et al. (2010) designed an independent scale for late-M dwarfs relying on model fits to high-resolution nearinfrared spectra. These authors adopted effective temperatures of $\sim 2930 \mathrm{~K}$ and $2850 \mathrm{~K}$ for USco 66AB (M6) and USco 100 (M7), respectively, in agreement with the scale designed by Luhman (1999) within current error bars. For later spectral types, we kept the scale proposed by Lodieu et al. (2008) i.e. $2250 \pm 50 \mathrm{~K}$ for $\mathrm{L} 0,2100 \pm 100 \mathrm{~K}$ for L1, and $1950 \pm 150 \mathrm{~K}$ for $\mathrm{L} 2$. The temperatures adopted in this paper are compiled in Table 3.

We also defined a set of bolometric corrections in the $J$-band (Luhman 1999) for the full range of spectral types covered by our study. For late-M and early-L dwarfs, we considered the values published by Dahn et al. (2002) and Vrba et al. (2004) whereas values for earlier spectral types come from Leggett et al. (2000). The latter bolometric correction are derived for old field dwarfs and may not be valid for younger brown dwarfs. The adopted mean values are compiled in the last row of Table 3.

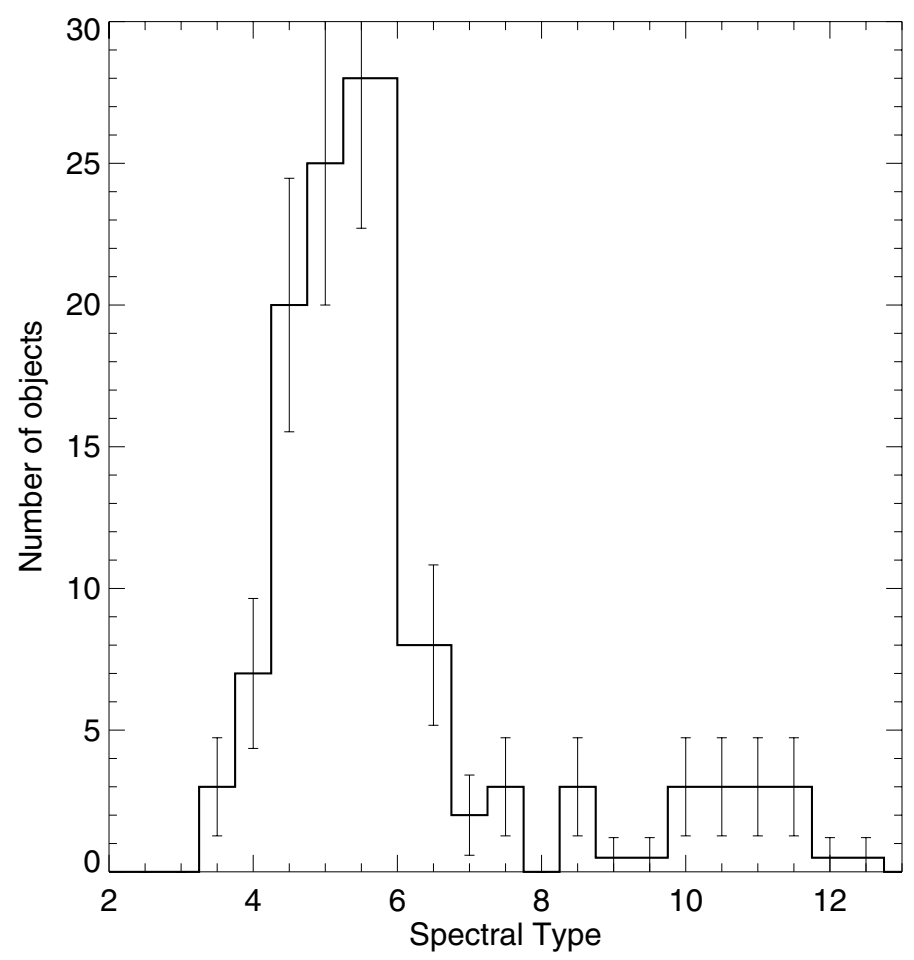

Fig. 7. Luminosity function or number of USco spectroscopic members as a function of spectral types ( $4 \equiv \mathrm{M} 4,5 \equiv \mathrm{M} 5$, etc.).

\subsection{The luminosity function}

For the sources in common between the optical and near-infrared spectroscopic follow-up, we have kept the spectral types derived from the optical spectra. Table 3 lists the numbers of sources per spectral type category. However, to plot the luminosity function, we need to create bins of equal size which we choose to be 0.5 subtype. Therefore, we put together the sources classified as M5.5 and M5.75 into the M5.5 bin, the objects with M6.25 and M6.5 into the M6.5 bin, and divided the numbers of sources in the M9, L0, L1, and L2 categories by two to split them up into 0.5 subtype bins. The resulting luminosity function i.e. the number of objects as a function of spectral type and binned by half a subclass, is shown in Fig. 7.

Although our sample in the region studied here is complete, the spectral type of every object is associated with an error on the spectral assignment. Hence, we have plotted error bars that correspond to the square root of the count in each bin. The number of objects increases quickly towards M5, peaks around M6 and then decreases swiftly to M7 where the M7/M8 gap is proposed due to the onset of dust in substellar atmospheres (Dobbie et al. 2002b). The luminosity function appears flat towards later spectral types and cooler temperatures. The overall trend is very similar to the analogous distribution for IC 348 (valid for spectral types earlier than M9) depicted in Fig. 11 of Luhman et al. (2003b).

With the effective temperatures and the bolometric luminosities derived for each individual object in our spectroscopic 
N. Lodieu et al.: Multi-fibre optical spectroscopy of low-mass stars and brown dwarfs in Upper Scorpius

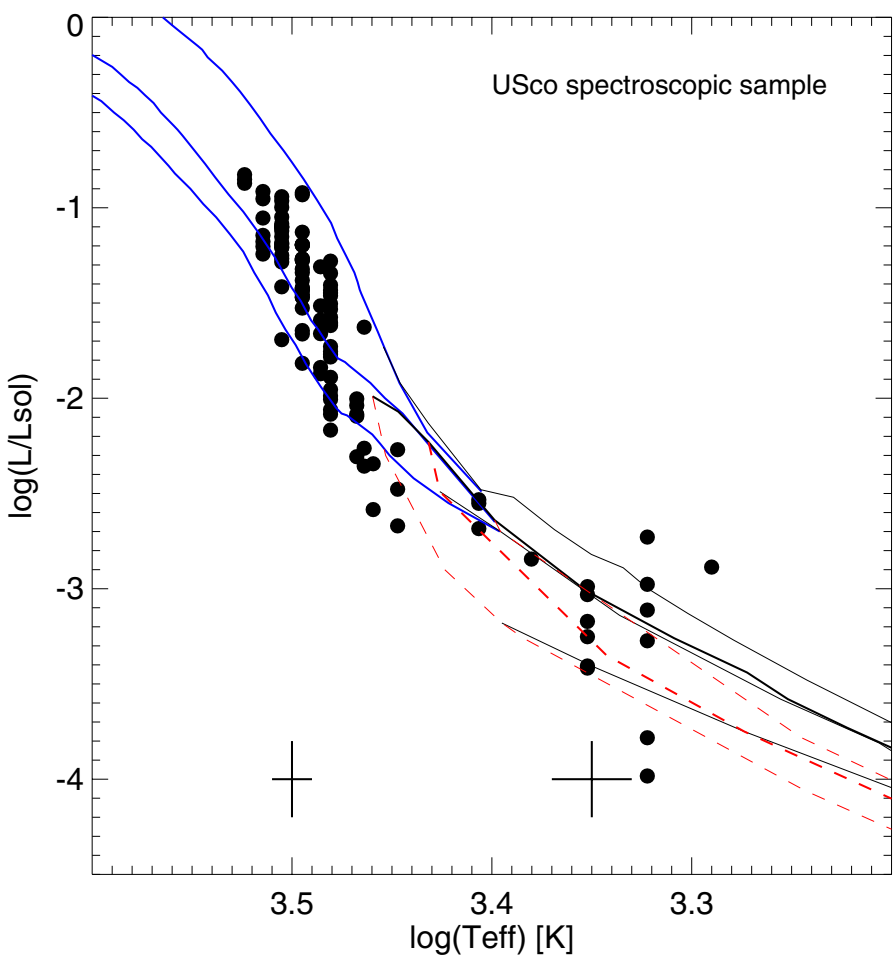

Fig. 8. Hertzsprung-Russell diagram for USco spectroscopic members confirmed at optical wavelengths with AAT/AAOmega in this paper and in the near-infrared with Gemini/GNIRS (Lodieu et al. 2008). Solid lines are NextGen and DUSTY isochrones at 1, 5, and 10 Myr. Dashed lines represent isomasses of $0.05,0.03$, and $0.02 M_{\odot}$ from the DUSTY models only (Chabrier et al. 2000). Typical error bars have been added at the bottom of the plot.

sample of confirmed USco members, we can plot an Hertzsprung-Russell diagram shown in Fig. 8. Our diagram is similar to Fig. 8 of Slesnick et al. (2008) which involves a larger number of members due to the larger areal coverage. The mean age of our sample is around $5 \mathrm{Myr}$ with a dispersion between 1 and $10 \mathrm{Myr}$, consistent with the discussion presented in Slesnick et al. (2008). Our survey extends to lower effective temperatures and luminosities after including the brown dwarfs confirmed spectroscopically in the near-infrared with Gemini (Lodieu et al. 2008). Mixing optical and near-infrared spectral types may introduce some biases at low masses and, thus, affect the determination of the mass function. Indeed, near-infrared spectral types tend to be later than optical spectral types (Lodieu et al. 2005b; Luhman et al. 2003b) by 1-2 subclass (but not always; e.g. USco J160648.18-223040.1), yielding cooler temperatures by $\sim 100 \mathrm{~K}$ and therefore lower masses.

\subsection{The mass function}

Several groups have developed models with different degrees of complexity to infer evolutionary tracks for young low-mass stars and brown dwarfs, e.g. Palla \& Stahler (1993), D'Antona \& Mazzitelli (1994), Burrows et al. (1997), Siess et al. (2000), Baraffe et al. (1998), Chabrier et al. (2000), and (Baraffe et al. 2002). We refer the reader to the study by Hillenbrand \& White (2004) for a detailed study of the discrepancies observed between models over a wide range of masses. For consistency with our earlier work in other regions targeted by the GCS (Lodieu et al. 2007a, 2009), we have derived the bolometric luminosities using the apparent $J$ magnitudes and the bolometric corrections listed in Table 3, assuming a bolometric magnitude of 4.74 for the Sun and a distance of $145 \mathrm{pc}$ for USco. Then to derive masses we have interpolated the luminosities provided by the NextGen (Baraffe et al. 1998) and DUSTY (Chabrier et al. 2000) for temperatures above and below $2500 \mathrm{~K}$, respectively.

Our sample contains 113 spectroscopic members with spectral types between M3.25 and L2 and masses between 0.4 and $0.006 M_{\odot}$ identified in a 6.5 square degree area surveyed by the UKIDSS GCS. However, we estimate our photometric survey to be complete only down to $0.01 M_{\odot}$. Only 20 objects of the original 129 photometric member candidates (Lodieu et al. 2007b) do not have spectra and are not included in the estimate of the mass function. The resulting mass function is shown in Fig. 9 along with its error bars (square root of the number of objects per mass bin) and compared to the spectroscopic mass function of Slesnick et al. (2008) created from a sample of 377 objects over the $\sim 0.6-0.02 M_{\odot}$ mass range spread over 150 square degrees (dashed line in Fig. 9). However the spectroscopic followup presented in Slesnick et al. (2008) is based on a $\sim 15 \%$ sampling, variable across the magnitude range, with a peak at around $r \sim 19.5 \mathrm{mag}$ and a sharp drop-off at fainter magnitudes. Therefore, we have tentatively scaled their spectroscopic mass function (thick line in Fig. 12 of Slesnick et al. 2008) to our areal coverage (22.5 vs. 6.5 square degrees represent a scaling factor of 3.46 in logarithmic units). We have also overplotted the mass function in IC 348 (dotted line in Fig. 9, Luhman et al. 2003b) without any scaling (the binning slightly differs from the USco mass function).

The first data point of our USco mass function is incomplete due to saturation of the GCS photometric survey. Our mass functions seems to peak at around $0.2 \pm 0.1 M_{\odot}$ but this should be taken with a grain of salt as our survey is incomplete at masses higher than $\sim 0.4 M_{\odot}$. If true however, the peak would be consistent with the characteristic mass found in the Pleiades (Moraux et al. 2003; Lodieu et al. 2007a), IC 348, the Trapezium (Luhman et al. 2000), and the field (Chabrier 2003) mass functions. After a decline occuring around $0.1 M_{\odot}$, our mass function flattens in the substellar regime. The decline seen beyond $0.01 M_{\odot}$ is due to the incompleteness of our photometric survey.

The overall shape of the USco mass function is similar to the mass function in IC 348 derived by Luhman et al. (2003b) over the stellar mass range and beyond the hydrogen-burning limit as it can be seen from the mass function (left-hand side plot of Fig. 9) and in the cumulative distribution (right-hand side plot of Fig. 9). The number of objects found by Slesnick et al. (2008) in the stellar regime is lower by a factor of two. One explanation for the discrepancy may be an underestimate of the incompleteness at the bright end of Slesnick et al. (2008)'s survey. The use of different theoretical isochrones to transform observables into masses is unlikely to account for such a large difference in the number of $0.3-0.1 M_{\odot}$ stars. We also find a larger number of brown dwarfs below $0.03 M_{\odot}$, indicating a poor sensitivity of Slesnick et al. (2008)'s optical survey to low-mass substellar objects.

The cumulative distributions shown in the right-hand side plot of Fig. 9 represent the normalised number of objects down to a given mass in logarithmic units. All mass functions appear similar within the error bars over the $0.3-0.06 M_{\odot}$ mass range as indicated by the mass functions. However, the cumulative mass functions plotted in the right-hand side panel of Fig. 9 show that the number of brown dwarfs is higher (although possibly within current error bars) in USco than IC 348 and the extrapolation of the field mass function in the common region where the USco and IC 348 surveys are complete. To quantify 

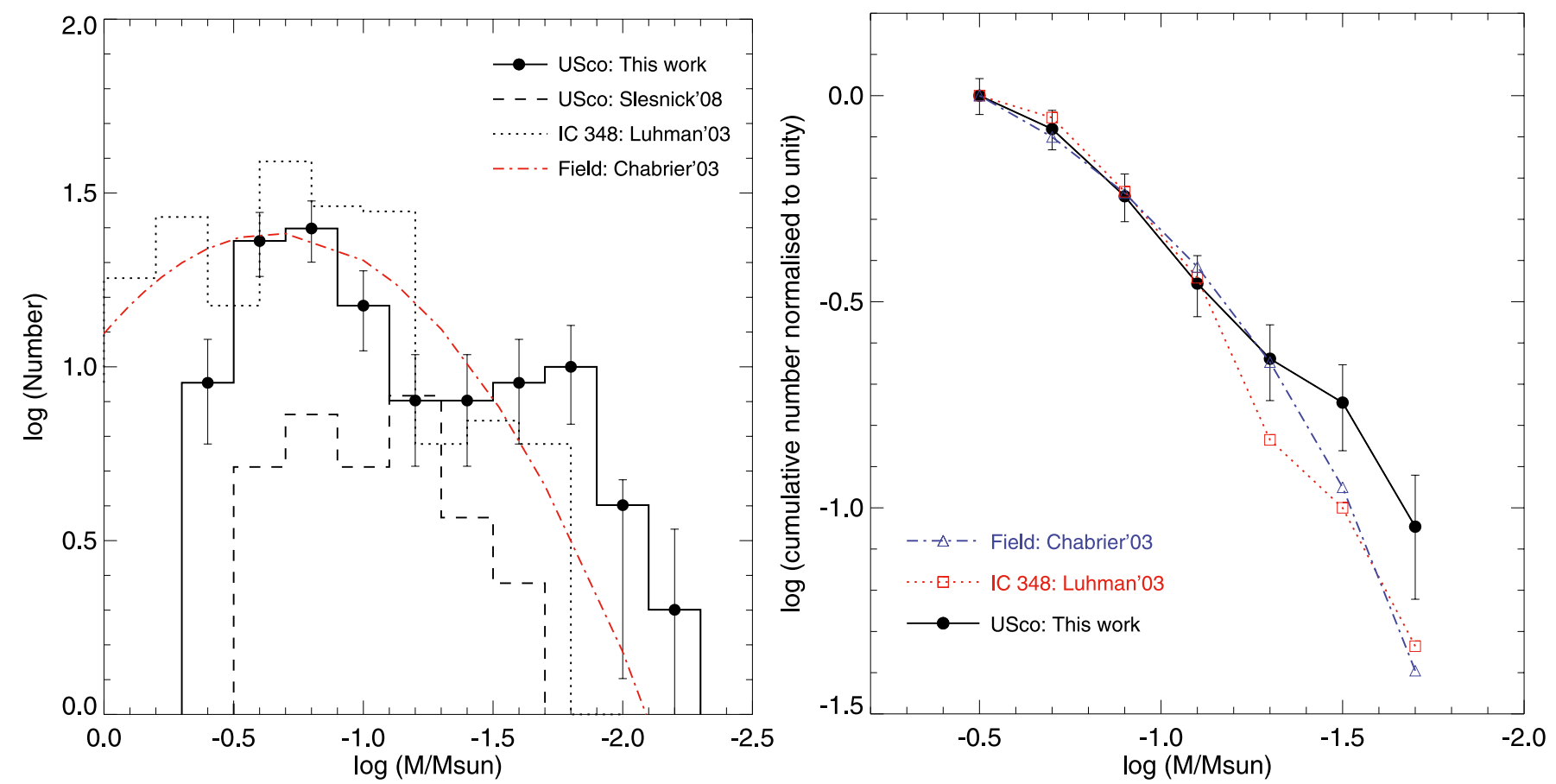

Fig. 9. Left: spectroscopic mass function for 6.5 square degree in the central region of USco valid between 0.35 and $0.01 M_{\odot}$. Overplotted as a dashed histogram is the spectroscopic mass function (corrected for incompleteness) published by Slesnick et al. (2008) and scaled to an area of 6.5 square degrees. The mass functions in IC 348 and the field are overplotted as a dotted histogram (Luhman et al. 2003b) and dot-dash line (Chabrier 2003), respectively. Right: cumulative distributions (logarithm of sum of the number of objects down to a given mass normalised to unity at the higher mass) for the mass functions in USco (filled dots and black line), IC 348 (open squares and dotted line), and the field (open triangles and dot-dahed line) over the mass range where the USco and IC 348 mass functions are complete. Error bars are included for the USco mass function only.

this hypothesis, we have computed the ratio of the number of stars $\left(M=0.35-0.08 M_{\odot}\right)$ and the number of brown dwarfs ( $M=0.08-0.02 M_{\odot}$ ) for IC 348 and USco. We find ratios of $21 / 104=0.20 \pm 0.03$ for IC 348 (Luhman et al. (2003b) finds $0.18 \pm 0.04$ for a slightly different definition of the star/brown dwarf ratio) and $24 / 59=0.41 \pm 0.03$ for USco, suggesting that USco may indeed contain an excess of substellar objects as originally suggested by Preibisch \& Zinnecker (2002) and corroborated by Slesnick et al. (2008). As such, the USco association would be the best place to search for the turn down of the mass function and investigate the issue of the fragmentation limit.

\section{Conclusions}

We have presented a multi-fibre spectroscopic follow-up of more than 100 photometric candidates identified in the central part of the USco association. The main results of our survey are:

- We have confirmed the spectroscopic membership of 90 photometric candidates based on the presence of $\mathrm{H} \alpha$ in emission and weak gravity-sensitive features in their optical spectra.

- We have rejected four photometric candidates as members, implying a completeness level of our original selection between 69 and $96 \%$.

- $\sim 10 \%$ of the new spectroscopic members show very strong $\mathrm{H} \alpha$ emission lines, indicating the presence of disks and accretion.

- We are able to detect lithium in absorption and the magnesium triplet in most members despite only moderate spectral resolution.

- We have demonstrated the efficiency of our original photometric and proper motion selections.
- Among the photometric non-members, we have identified three young disk-bearing sources showing signs of outflows.

- We have assigned tentative spectral types to a large number of stars falling in the AAOmega field of view.

- The luminosity drawn from a spectroscopic sample of 113 sources with spectral types ranging from M3.25 to L2 shows a peak at around M6 and is flat at later spectral types.

- The mass function derived in this 6.5 square degree area in Upper Sco is very similar to that derived of IC 348 and the extrapolation of the field mass function down to $\sim 0.06 M_{\odot}$.

- The mass function may peak around $0.2 M_{\odot}$ and is flat in the substellar regime.

- The number of low mass brown dwarfs in USco appears higher than in IC 348 although within current error bars, pointing towards the original suggestion that USco might be rather rich in low-mass brown dwarfs and, thus, the best place to search for the turn down of the mass function.

The low level of contamination inferred from our original photometric and proper motion selection using data taken during the science verification phase of the UKIDSS GCS implies that similar selection can be applied to the full association to carry out a global study of the region. The latest GCS data release includes $>30 \%$ coverage of the USco association. Subsequent data releases will soon complete the coverage in all filters and we will be able to identified new members to investigate important issues, including the distribution of low-mass stars and brown dwarfs with respect to massive stars and possible variation of the mass function within the association. The efficiency of the GCS is favorable to future deep surveys of USco aiming at finding cooler brown dwarfs and concentrating on the topic of the fragmentation limit. The advent of VISTA will also provide 
a complementary second epoch in USco to improve the astrometric selection.

Our work also has some implications for the study of other regions surveyed by the GCS. The combination of two optical filters $(Z, Y)$, three infrared bands, and proper motion has confirmed the power of the GCS in several regions, including the Pleiades, USco, and $\sigma$ Orionis. Other regions such as Orion and Taurus are included in the GCS and will provide important clues to address the issue of the universality of the IMF as a function of age and environment.

Acknowledgements. N.L. acknowledges funding from the Spanish Ministry of Science and Innovation through the Ramón y Cajal fellowship number 08-30301-02. N.L. also thanks the Anglo-Australian Observatory (AAO) for funding part of his stay at Epping in February/March 2010 and the AAO staff for an enjoyable visit. Based on data obtained with the AAOmega spectrograph installed on the Anglo Australian 3.9-m telescope in Siding Springs, Australia (program 07A/040). We thank Dr. Kevin Luhman for sending us the optical spectra of young M dwarfs and the data to plot the IMF of IC 348.

The United Kingdom Infrared Telescope is operated by the Joint Astronomy Centre on behalf of the UK Science Technology and Facility Council. This research has made use of the Simbad database, operated at the Centre de Données Astronomiques de Strasbourg (CDS), and of NASA's Astrophysics Data System Bibliographic Services (ADS).

\section{References}

Ardila, D., Martín, E., \& Basri, G. 2000, AJ, 120, 479

Baraffe, I., Chabrier, G., Allard, F. \& Hauschildt, P. H. 1998, A\&A, 337, 403

Baraffe, I., Chabrier, G., Allard, F., \& Hauschildt, P. H. 2002, A\&A, 382, 563

Barrado y Navascués, D., \& Martín, E. L. 2003, AJ, 126, 2997

Barrado y Navascués, D., Zapatero Osorio, M. R., Martín, E. L., et al. 2002, A\&A, 393, L85

Bouy, H., \& Martín, E. L. 2009, A\&A, 504, 981

Briceño, C., Hartmann, L., Stauffer, J., \& Martín, E. 1998, AJ, 115, 2074

Briceño, C., Luhman, K. L., Hartmann, L., Stauffer, J. R., \& Kirkpatrick, J. D. 2002, ApJ, 580, 317

Burrows, A., Marley, M., Hubbard, W. B., et al. 1997, ApJ, 491, 856

Chabrier, G. 2003, PASP, 115, 763

Chabrier, G., Baraffe, I., Allard, F., \& Hauschildt, P. 2000, ApJ, 542, 464

Dahn, C. C., Harris, H. C., Vrba, F. J., et al. 2002, AJ, 124, 1170

D'Antona, F., \& Mazzitelli, I. 1994, ApJS, 90, 467

de Bruijne, J. H. J., Hoogerwerf, R., Brown, A. G. A., Aguilar, L. A., \& de Zeeuw, P. T. 1997, in ESA SP-402: Hipparcos - Venice '97, 575

de Zeeuw, P. T., Hoogerwerf, R., de Bruijne, J. H. J., Brown, A. G. A., \& Blaauw, A. 1999, AJ, 117,354

Dobbie, P. D., Kenyon, F., Jameson, R. F., et al. 2002a, MNRAS, 329, 543

Dobbie, P. D., Pinfield, D. J., Jameson, R. F., \& Hodgkin, S. T. 2002b, MNRAS, 335, L79

Emerson, J. P. 2001, in The New Era of Wide Field Astronomy, ed. R. Clowes, A. Adamson, \& G. Bromage, ASP Conf. Ser., 232, 339

Haisch, K. E., Lada, E. A., \& Lada, C. J. 2001, AJ, 121, 2065

Herczeg, G. J., Cruz, K. L., \& Hillenbrand, L. A. 2009, ApJ, 696, 1589

Hillenbrand, L. A., \& Carpenter, J. M. 2000, ApJ, 540, 236

Hillenbrand, L. A., \& White, R. J. 2004, ApJ, 604, 741

Kirkpatrick, J. D., Henry, T. J., \& McCarthy, D. W. 1991, ApJS, 77, 417
Kunkel, M. 1999, Ph.D. Thesis, Julius-Maximilians-Universität Würzburg Lawrence, A., Warren, S. J., Almaini, O., et al. 2007, MNRAS, 379, 1599 Leggett, S. K., Allard, F., Dahn, C., et al. 2000, ApJ, 535, 965

Lewis, I. J., Cannon, R. D., Taylor, K., et al. 2002, MNRAS, 333, 279

Lodieu, N., McCaughrean, M. J., Barrado Y Navascués, D., Bouvier, J., \& Stauffer, J. R. 2005a, A\&A, 436, 853

Lodieu, N., Scholz, R.-D., McCaughrean, M. J., et al. 2005b, A\&A, 440, 1061

Lodieu, N., Hambly, N. C., \& Jameson, R. F. 2006, MNRAS, 373, 95

Lodieu, N., Dobbie, P. D., Deacon, N. R., et al. 2007a, MNRAS, 380, 712

Lodieu, N., Hambly, N. C., Jameson, R. F., et al. 2007b, MNRAS, 374, 372

Lodieu, N., Hambly, N. C., Jameson, R. F., \& Hodgkin, S. T. 2008, MNRAS, 383,1385

Lodieu, N., Zapatero Osorio, M. R., Rebolo, R., Martín, E. L., \& Hambly, N. C. 2009, A\&A, 505, 1115

Luhman, K. L. 1999, ApJ, 525, 466

Luhman, K. L. 2000, ApJ, 544, 1044

Luhman, K. L. 2004a, ApJ, 602, 816

Luhman, K. L. 2004b, ApJ, 617, 1216

Luhman, K. L. 2007, ApJS, 173, 104

Luhman, K. L. \& Steeghs, D. 2004, ApJ, 609, 917

Luhman, K. L., Rieke, G. H., Young, E. T., et al. 2000, ApJ, 540, 1016

Luhman, K. L., Briceño, C., Stauffer, J. R., et al. 2003a, ApJ, 590, 348

Luhman, K. L., Stauffer, J. R., Muench, A. A., et al. 2003b, ApJ, 593, 1093

Luhman, K. L., Allers, K. N., Jaffe, D. T., et al. 2007, ApJ, 659, 1629

Luhman, K. L., Hernández, J., Downes, J. J., Hartmann, L., \& Briceño, C. 2008, ApJ, 688, 362

Martín, E. L., Rebolo, R., \& Zapatero Osorio, M. R. 1996, ApJ, 469, 706

Martín, E. L., Delfosse, X., \& Guieu, S. 2004, AJ, 127, 449

Martin, E. L., Phan-Bao, N., Bessell, M., et al. 2010, A\&A

Mayne, N. J. \& Harries, T. J. 2010, MNRAS, 409, 1307

Miller, G. E. \& Scalo, J. M. 1979, ApJS, 41, 513

Miszalski, B., Shortridge, K., Saunders, W., Parker, Q. A., \& Croom, S. M. 2006, MNRAS, 371, 1537

Moraux, E., Bouvier, J., Stauffer, J. R., \& Cuillandre, J.-C. 2003, A\&A, 400, 891

Muench, A. A., Lada, E. A., Lada, C. J., \& Alves, J. 2002, ApJ, 573, 366

Palla, F., \& Stahler, S. W. 1993, ApJ, 418, 414

Preibisch, T., \& Zinnecker, H. 2002, AJ, 123, 1613

Preibisch, T., Guenther, E., Zinnecker, H., et al. 1998, A\&A, 333, 619

Preibisch, T., Guenther, E., \& Zinnecker, H. 2001, AJ, 121, 1040

Reid, W. A., \& Parker, Q. A. 2010, MNRAS

Riaz, B., Lodieu, N., \& Gizis, J. E. 2009, ApJ, 705, 1173

Rice, E. L., Barman, T., Mclean, I. S., Prato, L., \& Kirkpatrick, J. D. 2010, ApJS, 186,63

Salpeter, E. E. 1955, ApJ, 121, 161

Scalo, J. M. 1986, Fund. Cosm. Phys., 11, 1

Sharp, R., Saunders, W., Smith, G., et al. 2006, in Ground-based and Airborne Instrumentation for Astronomy, ed. I. S. McLean, \& M. Iye, Proc. SPIE, 6269, 62690G

Siess, L., Dufour, E., \& Forestini, M. 2000, A\&A, 358, 593

Slesnick, C. L., Hillenbrand, L. A., \& Carpenter, J. M. 2004, ApJ, 610, 1045

Slesnick, C. L., Carpenter, J. M., \& Hillenbrand, L. A. 2006, AJ, 131, 3016

Slesnick, C. L., Hillenbrand, L. A., \& Carpenter, J. M. 2008, ApJ, 688, 377

Turnshek, D. E., Turnshek, D. A., Craine, E. R., \& Boeshaar, P. C. 1985, An atlas of digital spectra of cool stars (Types G, K, M, S and C), ed. D. E. Turnshek, D. A. Turnshek, E. R. Craine, \& P. C. Boeshaar

Vrba, F. J., Henden, A. A., Luginbuhl, C. B., et al. 2004, AJ, 127, 2948

Walter, F. M., Vrba, F. J., Mathieu, R. D., Brown, A., \& Myers, P. C. 1994, AJ, 107,692

West, A. A., Hawley, S. L., Bochanski, J. J., et al. 2008, AJ, 135, 785

Zacharias, N., Finch, C., Girard, T., et al. 2010, AJ, 139, 2184

Pages 14 to 19 are available in the electronic edition of the journal at http://www . aanda.org 
A\&A 527, A24 (2011)

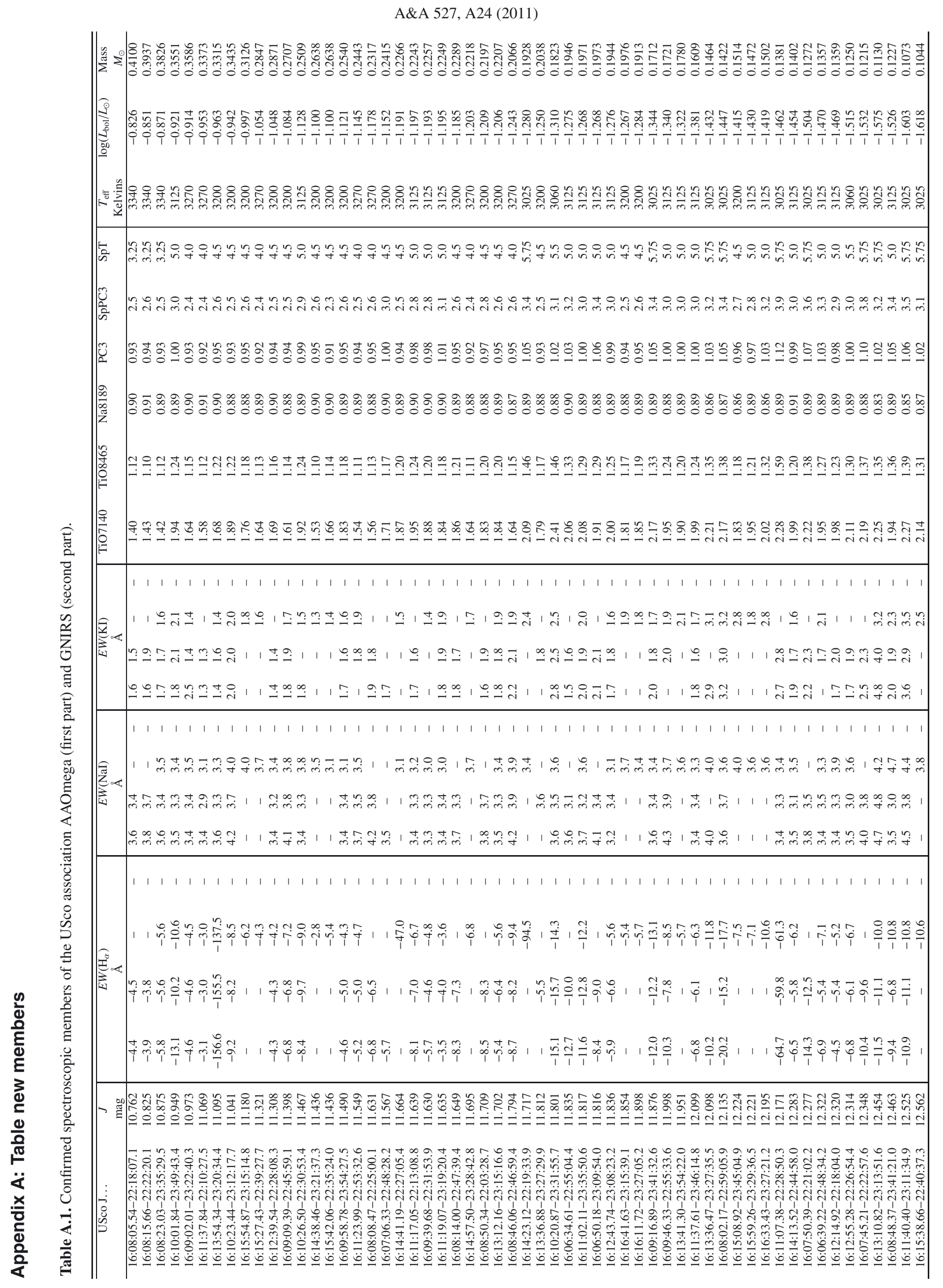

Page 14 of 19 
N. Lodieu et al.: Multi-fibre optical spectroscopy of low-mass stars and brown dwarfs in Upper Scorpius

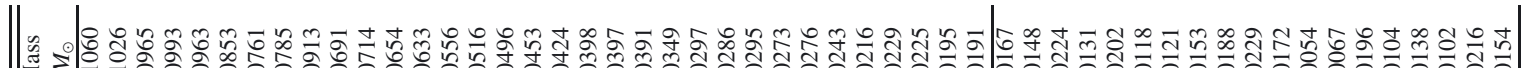

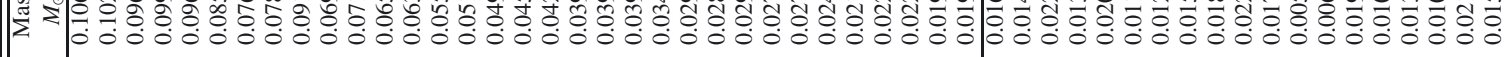

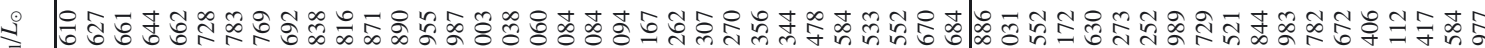

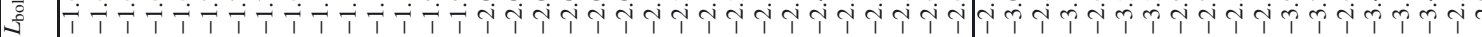

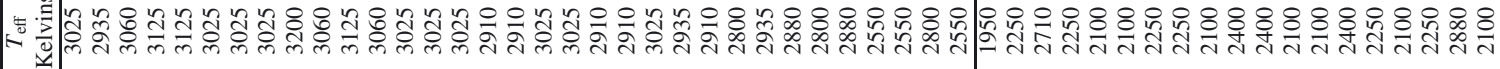

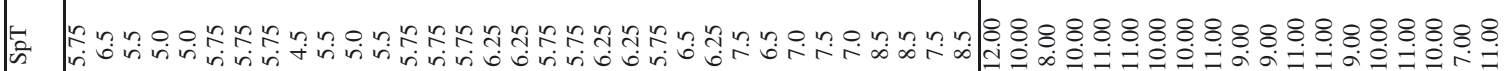

$\sum_{\substack{n \\ n}}^{n}$

$\tilde{2}$

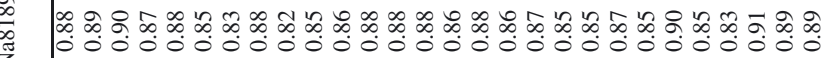

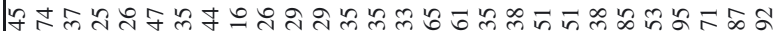

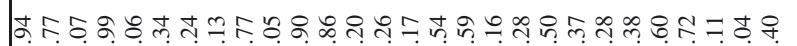

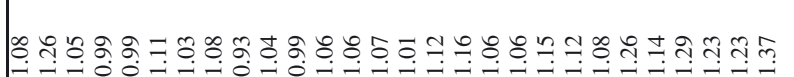

$\stackrel{0}{i=1}$

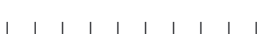

i

is

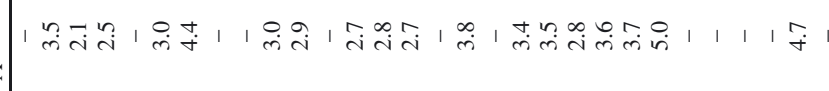

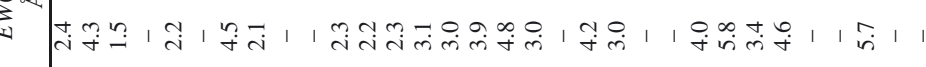

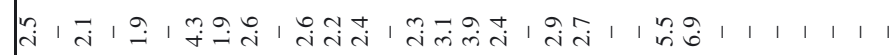

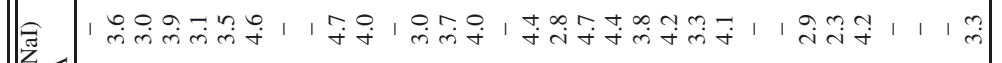

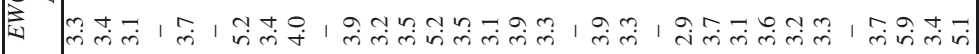

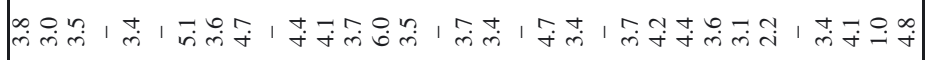

产

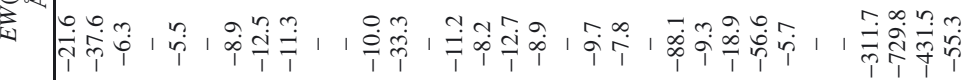

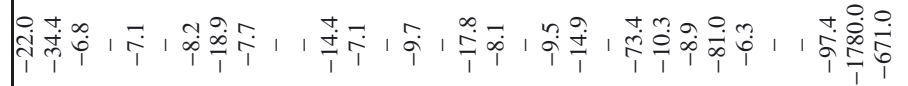

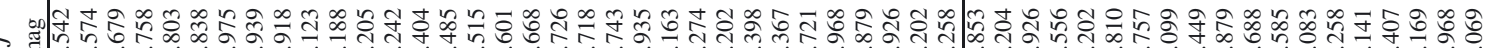

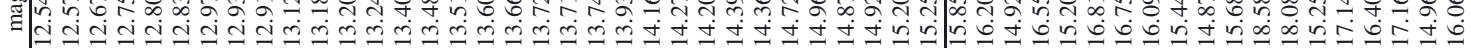
orma $=0$ -

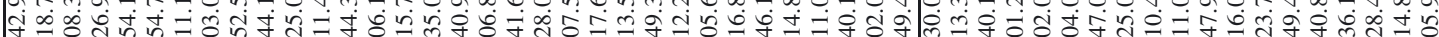

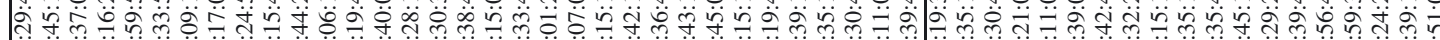

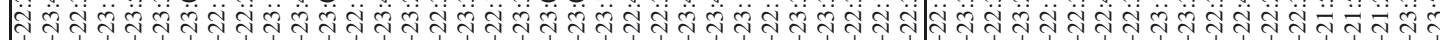
年

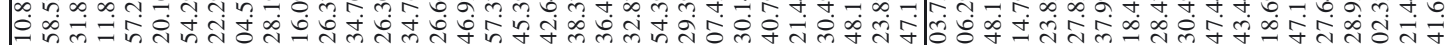

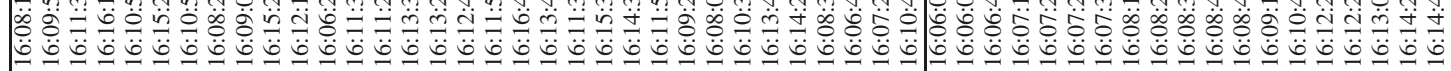

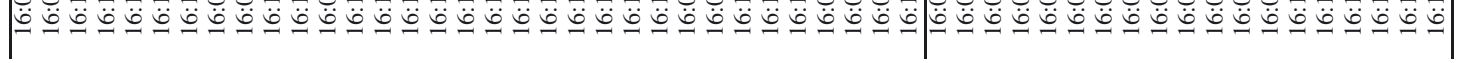




\section{Appendix B: Classification of stars in the AAOmega field}

The full sample of over 600 stars in the AAOmega field assigned to a fibre in addition to the USco member candidates is available electronically (Table B.1). An example of the table is provided below for guidance only. Columns 1 and 2 give the coordinates (J2000); Cols. 3-7 the ZY JHK photometry, Cols. 8-9 the proper motions (in mas/yr) in right ascension and declination, respectively; Col. 10 the spectral types based on the visual comparison with the templates listed below; and Col. 11 the name give prior to the fibre assignment.

This sample can be divided into several groups, each of them characterised by one template with a good quality spectrum (see Fig. B.2). Spectral types of M dwarfs should be accurate to half a subclass whereas others are tentative spectral types. The templates are:

- UGCS J160709.43-234031.8 or field6062 classified as $\mathrm{M} 1.5$;

- UGCS J160709.43-234031.8 or field3147 classified as M2;

- UGCS J160709.43-234031.8 or field4452 classified as $\mathrm{M} 2.5$;

- UGCS J160709.43-234031.8 or field2656 classified as M3$\mathrm{M} 3.5$;

- UGCS J160709.43-234031.8 or field1771 classified as M4;

- UGCS J160709.43-234031.8 or field6901 classified as M4.5;

- UGCS J160709.43-234031.8 or field1296 classified as M5.25-5.5;

- UGCS J160709.43-234031.8 or field633 classified as M5.75-M6;

- Four objects classified as dM6+ i.e. later than M6;

- UGCS J160634.44-231517.7 or field3830 is classified as a late-K/early-M;

- UGCS J160709.43-234031.8 or field2636 is classified as a late-K/early-M;

- UGCS J160733.42-224609.4 or field477 is classified as late-K/early-M and show a different level of reddening than field3830. The position of these objects in the $(H-K, J-H)$ colour-colour diagram (Fig. B.1) corroborate the classification as dwarfs rather than giants (e.g. Fig. 5 in Dobbie et al. 2002a). However, we can not rule our that some giants are included in this sample;

- UGCS J160544.57-231310.6 or field522 is classified as a late-K dwarf or giant;

- UGCS J160632.31-224817.2 or field1530 is classified as a late-G giant. Sources in this group show similar features but may have different levels of reddening;

- Two white dwarfs: UGCS J160757.34-231248.5 (field4930) and UGCS J160917.16-231020.1 (field1861);

- Three young active flare M dwarfs, see Sect. 5.4;

- 18 objects unclassified;

- 11 objects with no signal.

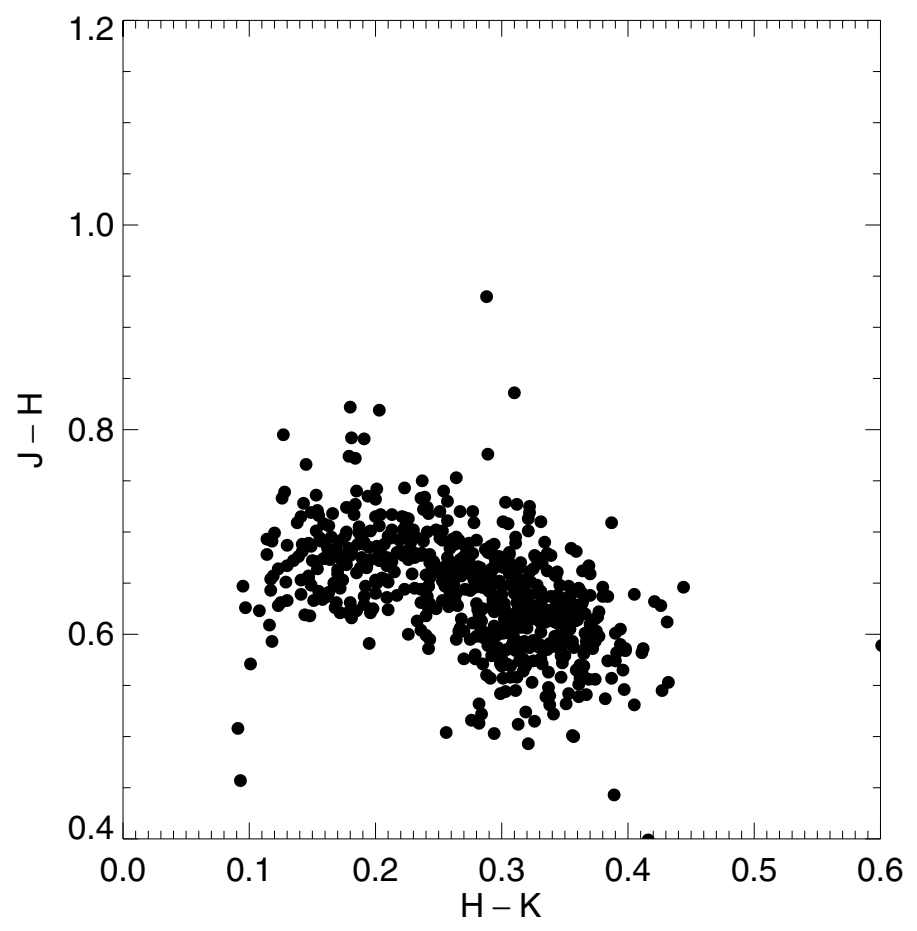

Fig. B.1. $(H-K, J-H)$ two-colour diagram for stars in the AAOmega field. 
N. Lodieu et al.: Multi-fibre optical spectroscopy of low-mass stars and brown dwarfs in Upper Scorpius
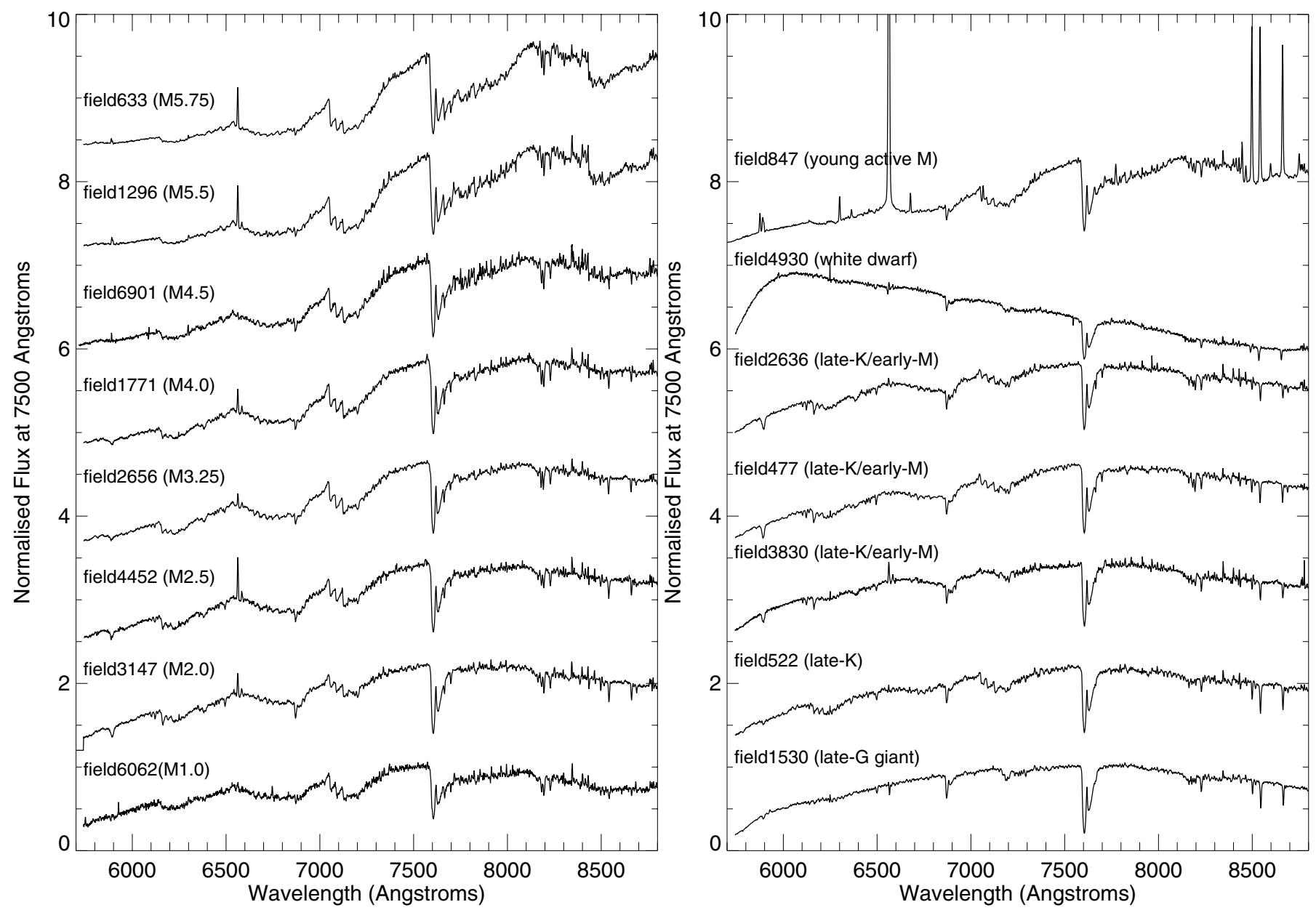

Fig. B.2. Example of optical spectra obtained with AAT/AAOmega for for stars in the AAOmega field not selected as photometric candidates. Spectra are normalised at $7500 \AA$ and offset for clarity. Left: a sample of field M dwarfs with spectral types ranging from M1 to M6. Right: a sample of late-K/early-M dwarfs, giants, white dwarfs, and young active $\mathrm{M}$ dwarfs.

Table B.1. Coordinates (J2000), ZYJHK photometry, and proper motions (in arcsec/yr) of stars in the AAOmega field ordered by increasing $Z$ magnitude.

\begin{tabular}{ccccccccccc}
\hline \hline $\begin{array}{c}\text { RA } \\
\text { h s m }\end{array}$ & $\begin{array}{c}\text { Dec } \\
\text { ○, }\end{array}$ & $\begin{array}{c}Z \\
\mathrm{mag}\end{array}$ & $\begin{array}{c}Y \\
\mathrm{mag}\end{array}$ & $\begin{array}{c}J \\
\mathrm{mag}\end{array}$ & $\begin{array}{c}H \\
\mathrm{mag}\end{array}$ & $\begin{array}{c}K \\
\mathrm{mag}\end{array}$ & $\begin{array}{c}\mu_{\alpha} \cos \delta \\
\mathrm{mas} / \mathrm{yr}\end{array}$ & $\begin{array}{c}\mu \delta \\
\mathrm{mas} / \mathrm{yr}\end{array}$ & SpT & Name \\
\hline $16: 07: 03.10$ & $-23: 31: 46.3$ & 12.024 & 11.539 & 10.982 & 10.393 & 9.792 & 10.21 & -24.90 & M3-M3.5 & field229 \\
$\ldots$ & $\ldots$ & $\ldots$ & $\ldots$ & $\ldots$ & $\ldots$ & $\ldots$ & $\ldots$ & $\ldots$ & $\ldots$ & $\ldots$ \\
$\ldots$ & $\ldots$ & $\ldots$ & $\ldots$ & $\ldots$ & $\ldots$ & $\ldots$ & $\ldots$ & $\ldots$ & $\ldots$ & $\ldots$ \\
$16: 15: 28.01$ & $-23: 08: 09.6$ & 17.489 & 16.816 & 16.263 & 15.698 & 15.339 & -67.67 & 57.84 & M5.25-M5 & field31105 \\
\hline
\end{tabular}


A\&A 527, A24 (2011)

\section{Appendix C: Proper-motion non-members}

Table C.1. Proper motion non members from Lodieu et al. (2007b) observed during our AAOmega spectroscopic follow-up.

\begin{tabular}{|c|c|c|c|c|c|c|c|c|c|c|}
\hline USco J... & $\begin{array}{c}Z \\
\text { mag }\end{array}$ & $\begin{array}{c}\mu_{\alpha} \cos \delta \\
\text { mas } / \mathrm{yr}\end{array}$ & $\begin{array}{c}\mu_{\delta} \\
\text { mas/yr }\end{array}$ & SpT & $\begin{array}{c}\mathrm{H} \alpha E W \\
\AA\end{array}$ & $\begin{array}{c}\mathrm{NaI} E W \\
\AA\end{array}$ & $\mathrm{Li}$ & PM & Memb & $\begin{array}{c}\text { UCAC PM } \\
\mathrm{mas} / \mathrm{yr}\end{array}$ \\
\hline $160827.38-221729.4$ & 12.76 & +0.38 & -8.08 & M5.0 & -9.2 & $1.82 .0(3.8)$ & $\bar{Y}$ & $\geq 2 \sigma$ & Memb & $-10.6-19.4$ \\
\hline 160 & 13.77 & 7.13 & -5.24 & M4.5 & 2.2 & 1.61 .9 & Y? & $\geq 2 \sigma$ & b & - \\
\hline $16090^{\circ}$ & 13.44 & 7.08 & -3.69 & M5.5 & .7 & 1.52 .0 & Y? & $\geq 2 \sigma$ & Memb & - \\
\hline 160908. & 14.12 & -36.50 & -29.63 & M5.75 & -17.4 & $2.22 .6(4.8)$ & $Y ?$ & $\geq 2.5 \sigma$ & Memb & - \\
\hline $161000.23-231219.4$ & 15.95 & -33.51 & -14.21 & M6.0 & -8.5 & $2.83 .4(6.2)$ & Y & $\geq 2 \sigma$ & NM & - \\
\hline-233109.0 & 14.84 & -5.10 & -2.45 & M5.75 & -10.0 & $1.31 .8(3.1)$ & $\mathrm{Y}$ & $\geq 2 \sigma$ & Memb & - \\
\hline $161412.41-221913.3$ & 12.47 & +8.80 & +0.24 & M4.5 & -7.0 & $1.61 .7(3.3)$ & $\mathrm{Y}$ & $\geq 3 \sigma$ & Memb & $-9.1-23.8$ \\
\hline $6.07-234510.5$ & 13.59 & -11.05 & +3.86 & M5.0 & -13.5 & $1.61 .9(3.5)$ & $\mathrm{Y}$ & $\geq 2.5 \sigma$ & Memb & - \\
\hline $161538.48-234156.1$ & 13.40 & -32.06 & -16.17 & M5.0 & -8.4 & $1.82 .1(3.9)$ & Y & $\geq 2 \sigma$ & Memb & - \\
\hline 161620.16-234414.4 & 12.08 & -33.21 & -11.33 & M5.0 & -9.8 & $1.82 .2(4.0)$ & $\mathrm{Y}$ & $\geq 2.5 \sigma$ & Memb & $-12.2-27.4$ \\
\hline
\end{tabular}


N. Lodieu et al.: Multi-fibre optical spectroscopy of low-mass stars and brown dwarfs in Upper Scorpius

\section{Appendix D: Candidates with proper motion and equivalent widths satisfying membership}

Table D.1. List of 50 candidates with proper motion within $3 \sigma$ from the cluster mean motion and with equivalent widths measurements of $\mathrm{H} \alpha$ and the Na I doublet consistent with membership.

\begin{tabular}{|c|c|c|c|c|c|c|c|c|c|c|c|}
\hline $\begin{array}{l}\text { RA } \\
\mathrm{h} \mathrm{m} \mathrm{s}\end{array}$ & $\begin{array}{l}\text { Dec } \\
0,11\end{array}$ & $\begin{array}{c}Z \\
\mathrm{mag}\end{array}$ & $\begin{array}{c}Y \\
\mathrm{mag}\end{array}$ & $\begin{array}{c}J \\
\mathrm{mag}\end{array}$ & $\begin{array}{c}H \\
\mathrm{mag}\end{array}$ & $\begin{array}{c}K \\
\mathrm{mag}\end{array}$ & $\begin{array}{c}\mu_{\alpha} \cos \delta \\
\text { mas } / \mathrm{yr}\end{array}$ & $\begin{array}{c}\mu_{\delta} \\
\mathrm{mas} / \mathrm{yr}\end{array}$ & $\begin{array}{c}\mathrm{H} \alpha E W \\
\AA\end{array}$ & $\begin{array}{c}\mathrm{Na} \mathrm{I} E W \\
\AA\end{array}$ & SpT \\
\hline $16: 07: 29.59$ & $-23: 08: 22.4$ & 12.815 & 12.365 & 11.749 & 10.970 & 10.188 & -30.12 & -29.77 & -158 & 5.1 & M3.0 \\
\hline $16: 14: 50.31$ & $-23: 32: 40.0$ & 12.758 & 12.218 & 11.605 & 10.803 & 10.067 & -18.21 & -16.37 & -108 & 3.0 & M4.5 \\
\hline $16: 05: 54.13$ & $-22: 57: 20.1$ & 13.720 & 13.392 & 13.008 & 12.337 & 12.124 & -17.98 & 2.37 & -2.9 & 3.3 & K7.0 \\
\hline 16:05:59.16 & $-23: 13: 30.3$ & 14.796 & 14.376 & 13.891 & 13.242 & 12.980 & -20.93 & -19.70 & -7.4 & 4.2 & M4.0 \\
\hline $16: 06: 21.43$ & $-23: 06: 39.7$ & 14.434 & 14.052 & 13.624 & 12.952 & 12.708 & -2.58 & -22.89 & -2.5 & 3.7 & M2.0 \\
\hline 16:06:33.11 & $-22: 39: 07.6$ & 14.318 & 14.015 & 13.579 & 12.942 & 12.701 & 5.08 & -7.73 & -8.2 & 3.5 & M2.0 \\
\hline $16: 06: 40.45$ & $-22: 24: 55.0$ & 14.935 & 14.467 & 13.972 & 13.307 & 13.005 & -0.63 & -1.36 & -14.5 & 4.9 & M4.0 \\
\hline $16: 06: 42.49$ & $-22: 47: 14.7$ & 15.664 & 15.159 & 14.666 & 14.065 & 13.759 & 4.42 & -6.36 & -22.8 & 5.0 & M4.0 \\
\hline $16: 06: 47.21$ & $-22: 24: 38.6$ & 15.325 & 14.829 & 14.292 & 13.672 & 13.358 & -14.01 & -2.72 & -18.9 & 4.5 & M3.2 \\
\hline $16: 06: 55.26$ & $-22: 47: 09.0$ & 16.083 & 15.555 & 15.053 & 14.436 & 14.110 & -8.30 & -0.35 & -34.4 & 4.6 & M5.5 \\
\hline 16:07:03.05 & $-23: 31: 46.2$ & 12.024 & 11.539 & 10.982 & 10.393 & 9.792 & 10.21 & -24.90 & -12.5 & 3.5 & M3.2 \\
\hline 16:07:06.86 & $-22: 52: 21.9$ & 15.233 & 14.776 & 14.307 & 13.634 & 13.367 & -34.77 & -42.90 & -6.3 & 4.2 & M4.0 \\
\hline 16:07:11.28 & $-23: 01: 10.8$ & 15.803 & 15.298 & 14.821 & 14.221 & 13.924 & 2.35 & -14.59 & -19.9 & 4.6 & M4.5 \\
\hline $16: 07: 23.23$ & $-23: 42: 24.5$ & 15.055 & 14.620 & 14.156 & 13.513 & 13.238 & -26.84 & -26.08 & -2.1 & 3.1 & M3.2 \\
\hline $16: 07: 24.27$ & $-23: 02: 27.4$ & 15.611 & 15.080 & 14.574 & 14.017 & 13.726 & -16.65 & -16.85 & -19.7 & 4.5 & M4.5 \\
\hline $16: 07: 39.59$ & $-23: 12: 13.6$ & 14.472 & 14.101 & 13.632 & 12.953 & 12.716 & 1.26 & -18.52 & -2.0 & 3.5 & M2.0 \\
\hline $16: 07: 41.59$ & $-23: 39: 09.7$ & 15.503 & 15.008 & 14.542 & 13.939 & 13.673 & 7.17 & -17.27 & -2.2 & 3.8 & M4.0 \\
\hline 16:07:46.74 & $-22: 25: 04.6$ & 15.416 & 14.955 & 14.467 & 13.852 & 13.584 & 6.87 & -19.85 & -13.5 & 4.7 & M4.0 \\
\hline 16:07:48.11 & $-22: 45: 29.8$ & 14.196 & 13.817 & 13.403 & 12.722 & 12.498 & -1.77 & -20.76 & -1.4 & 3.2 & M0.0 \\
\hline $16: 07: 49.13$ & $-22: 30: 42.3$ & 15.166 & 14.738 & 14.240 & 13.632 & 13.338 & -12.26 & -10.90 & -11.8 & 4.0 & M4.0 \\
\hline 16:07:53.62 & $-23: 41: 27.4$ & 15.528 & 15.008 & 14.535 & 13.854 & 13.565 & -10.06 & -1.51 & -1.3 & 4.9 & M4.0 \\
\hline $16: 07: 57.23$ & $-22: 31: 06.0$ & 15.178 & 14.743 & 14.261 & 13.613 & 13.313 & -25.67 & -18.50 & -10.0 & 4.1 & M4.0 \\
\hline 16:08:09.60 & $-22: 27: 25.8$ & 14.549 & 14.180 & 13.749 & 13.088 & 12.873 & -2.21 & -1.00 & -3.6 & 2.9 & M0.0 \\
\hline 16:08:10.35 & $-22: 54: 55.4$ & 15.397 & 14.909 & 14.446 & 13.786 & 13.503 & -0.28 & 2.79 & -10.8 & 4.0 & M3.2 \\
\hline 16:08:11.54 & $-22: 34: 52.5$ & 15.880 & 15.406 & 14.894 & 14.337 & 14.036 & -4.58 & -7.65 & -20.6 & 4.3 & M4.5 \\
\hline $16: 08: 23.42$ & $-22: 35: 04.5$ & 15.083 & 14.683 & 14.191 & 13.527 & 13.282 & 4.18 & -7.07 & -4.9 & 3.5 & M2.5 \\
\hline $16: 08: 26.24$ & $-22: 49: 51.9$ & 14.963 & 14.514 & 14.051 & 13.350 & 13.097 & -1.15 & -6.07 & -3.7 & 4.0 & M2.5 \\
\hline 16:08:40.97 & $-22: 46: 32.6$ & 15.307 & 14.785 & 14.282 & 13.630 & 13.343 & 5.34 & -8.09 & -9.7 & 4.8 & M4.0 \\
\hline 16:08:41.92 & $-23: 08: 02.7$ & 13.128 & 12.748 & 12.314 & 11.614 & 11.373 & -30.05 & -31.33 & 0.0 & 4.4 & M2.0 \\
\hline $16: 08: 47.94$ & $-23: 24: 19.3$ & 15.905 & 15.414 & 14.858 & 14.294 & 13.977 & -4.41 & -34.91 & -1.8 & 4.7 & M4.0 \\
\hline $16: 08: 52.43$ & $-22: 17: 59.0$ & 13.817 & 13.479 & 13.068 & 12.432 & 12.223 & -3.45 & -0.39 & -1.0 & 3.8 & M0.0 \\
\hline 16:08:53.34 & $-23: 07: 41.5$ & 15.516 & 15.046 & 14.506 & 13.900 & 13.580 & -1.96 & -18.55 & -5.0 & 4.6 & M4.0 \\
\hline 16:08:53.81 & $-22: 35: 01.5$ & 14.008 & 13.620 & 13.166 & 12.522 & 12.299 & 5.34 & -25.03 & -1.7 & 3.7 & M3.2 \\
\hline 16:08:58.04 & $-22: 43: 04.2$ & 15.478 & 14.942 & 14.463 & 13.816 & 13.506 & 3.09 & -5.86 & -14.1 & 4.3 & M4.5 \\
\hline 16:08:58.07 & $-22: 17: 52.2$ & 15.475 & 14.971 & 14.482 & 13.893 & 13.545 & -6.81 & -19.49 & -8.5 & 4.1 & M4.0 \\
\hline 16:09:03.40 & $-22: 16: 45.4$ & 14.418 & 14.062 & 13.646 & 12.971 & 12.762 & -28.06 & -21.50 & -3.6 & 3.5 & M0.0 \\
\hline 16:09:07.21 & $-22: 21: 15.8$ & 14.257 & 13.837 & 13.359 & 12.670 & 12.409 & -33.04 & -19.10 & -2.1 & 4.7 & M3.2 \\
\hline 16:09:07.81 & $-22: 21: 05.7$ & 16.974 & 16.383 & 15.840 & 15.227 & 14.912 & 3.01 & -24.30 & -31.4 & 4.0 & M4.5 \\
\hline 16:09:12.01 & $-23: 00: 57.3$ & 16.119 & 15.578 & 15.100 & 14.482 & 14.156 & -27.23 & -14.46 & -7.5 & 3.9 & M3.2 \\
\hline 16:09:15.41 & $-22: 59: 01.8$ & 16.090 & 15.528 & 15.034 & 14.424 & 14.079 & -24.41 & -22.02 & -8.4 & 4.7 & M4.0 \\
\hline 16:09:16.66 & $-23: 54: 48.6$ & 14.891 & 14.330 & 13.824 & 13.145 & 12.868 & -18.43 & 2.49 & -8.9 & 4.6 & M4.5 \\
\hline 16:09:47.50 & $-22: 15: 41.0$ & 15.888 & 15.392 & 14.886 & 14.267 & 13.985 & -0.38 & -44.42 & -5.2 & 4.7 & M4.5 \\
\hline 16:10:03.71 & $-22: 41: 34.6$ & 16.113 & 15.514 & 15.014 & 14.428 & 14.094 & -4.66 & -49.18 & -4.9 & 5.0 & M4.0 \\
\hline $16: 10: 10.76$ & $-23: 08: 27.3$ & 14.520 & 14.132 & 13.661 & 12.937 & 12.696 & -14.65 & 3.86 & -1.8 & 3.8 & M2.0 \\
\hline $16: 10: 13.43$ & $-22: 43: 41.7$ & 15.763 & 15.197 & 14.690 & 14.103 & 13.746 & 0.00 & 0.00 & -2.1 & 4.4 & M4.5 \\
\hline $16: 10: 55.38$ & $-23: 19: 53.5$ & 15.374 & 14.902 & 14.405 & 13.752 & 13.474 & -4.53 & -0.06 & -6.7 & 4.4 & M4.0 \\
\hline $16: 12: 57.88$ & $-23: 58: 26.5$ & 16.893 & 16.210 & 15.696 & 15.019 & 14.680 & -17.90 & -26.02 & -4.3 & 4.6 & M4.5 \\
\hline 16:14:02.69 & $-22: 57: 26.1$ & 14.820 & 14.300 & 13.815 & 13.176 & 12.876 & -11.13 & -10.24 & -2.0 & 4.8 & M3.2 \\
\hline $16: 15: 12.62$ & $-23: 56: 14.6$ & 14.299 & 13.813 & 13.346 & 12.693 & 12.378 & 3.70 & -10.74 & -4.8 & 3.9 & M3.2 \\
\hline $16: 16: 03.50$ & $-23: 41: 03.6$ & 15.156 & 14.572 & 14.063 & 13.441 & 13.147 & -2.97 & -10.99 & -6.4 & 4.9 & M4.5 \\
\hline $16: 16: 52.66$ & $-22: 29: 48.8$ & 16.014 & 15.431 & 14.853 & 14.268 & 13.932 & -19.27 & -10.57 & -8.6 & 4.6 & M4.5 \\
\hline $16: 16: 54.25$ & $-23: 56: 53.4$ & 16.712 & 16.081 & 15.558 & 14.879 & 14.543 & -10.83 & -5.65 & -3.2 & 4.8 & M4.5 \\
\hline
\end{tabular}

Notes. The eight proper motion non members reclassified as candidate members and listed in Table C.1 are not included. We have added at the top of the table the two accreting sources with proper motions consistent with USco for consistency. 